\title{
Genome-wide mapping of Vibrio cholerae VpsT binding identifies a mechanism for c-di-GMP homeostasis
}

Thomas Guest $^{1}$, James R.J. Haycocks ${ }^{1}$, Gemma Z.L. Warren ${ }^{1}$ and David C. Grainger ${ }^{1 *}$

${ }^{1}$ School of Biosciences, University of Birmingham, Edgbaston, Birmingham B15 2TT, UK.

email: d.grainger@bham.ac.uk

Tel: +44(0)121 4145437 


\begin{abstract}
Many bacteria use cyclic dimeric guanosine monophosphate (c-di-GMP) to control changes in lifestyle. The molecule, synthesised by proteins having diguanylate cyclase activity, is often a signal to transition from motile to sedentary behaviour. In Vibrio cholerae, c-di-GMP can exert its effects via the transcription factors VpsT and VpsR. Together, these proteins activate genes needed for $V$. cholerae to form biofilms. In this work, we have mapped the genome-wide distribution of VpsT in a search for further regulatory roles. We show that VpsT binds 23 loci and recognises a degenerate DNA palindrome having the consensus 5'-W. ${ }_{5} R_{-4}[C G]_{-3} Y_{-2} W_{-1} W_{+1} R_{+2}[G C]_{+3} Y_{+4} W_{+5}-3$ '. Most genes targeted by VpsT encode functions related to motility, biofilm formation, or c-di-GMP metabolism. Most notably, VpsT activates expression of the $v p v A B C$ operon that encodes a diguanylate cyclase. This creates a positive feedback loop needed to maintain intracellular levels of c-di-GMP. Mutation of the key VpsT binding site, upstream of $v p v A B C$, severs the loop and c-di-GMP levels fall accordingly. Hence, as well as relaying the c-di-GMP signal, VpsT impacts c-di-GMP homeostasis.
\end{abstract}

\title{
INTRODUCTION
}

Cholera is an acute diarrhoeal disease caused by the bacterium Vibrio cholerae (1). Globally, the current seventh pandemic is caused by strains of the $\mathrm{O} 1 \mathrm{El}$ Tor biotype that emerged in the Bay of Bengal, later spreading across the globe in three waves (2). Once introduced at a locality, $V$. cholerae strains capable of causing disease outbreaks can remain for several decades $(3,4)$. In part, this is because $V$. cholerae can spend long periods in aquatic environments (5). In this niche, $V$. cholerae forms biofilms on the chitinous exoskeletons of crustaceans and marine microbiota (5). Hence, these abundant surfaces are exploited as a source of carbon and nitrogen $(6,7)$. Major components of the $V$. cholerae biofilm matrix include vibrio polysaccharide (VPS) and three proteins; RbmA, RbmC and Bap-1 (8). Synthesis of VPS depends on the vps-I and vps-II gene clusters that are transcribed from the vpsA and $v p s L$ promoters respectively. The nearby $r b m$ gene cluster encodes RbmA and RbmB whilst Bap-1 is encoded elsewhere (9). Phase variation between smooth and rugose morphotypes also plays a role (10). Notably, rugose cells form biofilms more readily, present as corrugated colonies on agar, and aggregate in liquid culture $(11,12)$. 
In many bacteria, the decision to form biofilms involves the nucleotide second messenger cyclic dimeric guanosine monophosphate (c-di-GMP) (13). Within cells, c-di-GMP is produced by diguanylate cyclase (DGC) proteins. Such enzymes are identifiable by virtue of a "GGDEF" active site amino acid motif (14). Phosphodiesterases, with "EAL" or "HD-GYP" domains, degrade cdi-GMP (15). The signalling molecule is detected by factors that modulate cell behaviour. For instance, c-di-GMP controlled transcription factors can alter global patterns of gene expression (13). Similarly, the message can be relayed by riboswitches and sRNA molecules (16-18). In V. cholerae, two c-di-GMP dependent regulators, VpsR and VpsT, activate the vps-I, vps-II and rbm gene clusters (19-21). In rugose phase variants, VpsR and VpsT are required to maintain the phenotype $(22,23)$. The wider role of these regulators in the direct control of gene expression is less well understood.

First described in 2004, VpsT is a LuxR-type response regulator (20, 22, 24). Direct interaction with c-di-GMP is required for VpsT dimerisation and DNA binding (20). At the vpsA, vpsL and rbm promoters, VpsT displaces the histone-like nucleoid structuring (H-NS) protein that otherwise silences transcription (25). Conversely, at other loci, the transcription activation mechanism is unknown $(26,27)$. Transcriptional repression can also be mediated by VpsT. For instance, gene expression is blocked by VpsT binding across the rpoS transcription start sites (TSSs) (28). Similarly, VpsT can inhibit transcription of the divergent VC1303 and VC1304 genes (29). The DNA site bound by VpsT is called the T-box (30). Efforts to define a consensus T-box have been hampered by the paucity and variability of known VpsT targets. Initial studies revealed recognition of identical sequences by VpsT at the vpsL and vpsA promoters $(25,30)$. However, this sequence is not found at other targets $(26,27,30)$. In this work we sought a better understanding of VpsT, its DNA regulatory targets, and their interplay with c-di-GMP signalling.

\section{RESULTS AND DISCUSSION}

Genome-wide DNA binding by VpsT in Vibrio cholerae

We used ChIP-seq to map the binding of VpsT across the V. cholerae genome. To facilitate this, $v p s T$ was cloned in plasmid pAMNF. The resulting DNA construct, pAMNF-vpsT, encodes an N-terminal 3xFLAG-vpsT fusion that was used to transform $V$. cholerae strain E7946. Importantly, the amount of 3xFLAG-VpsT produced from pAMNF-vpsT (Figure S1, lanes 4-6) is similar to the 
amount of VpsT generated from the native chromosomal locus in both smooth (lanes 1-3) and rugose (lanes 7-9) cells. In subsequent ChIP-seq analysis, anti-FLAG antibodies were used to isolate regions of DNA bound to VpsT. An overview of the data are shown in Figure 1a. Genes are shown as dark blue lines (outer two tracks) and the VpsT binding signal is in cyan (inner track). Strikingly, VpsT does not bind chromosome II. Conversely, we identified 23 binding peaks for VpsT across chromosome I (Table 1). In previous work, VpsT binding at 7 different promoter regions has been shown in vitro $(25-28,30)$. We recovered 5 of these interactions (adjacent to vpsU, vpsA, vpsL, rbmA and rpoS) in our ChIP-seq analysis (Figure 1b, Table 1). We did not detect VpsT binding upstream of $\operatorname{tag}$ or $k a t B(26,27)$. Of the 23 ChIP-seq peaks for VpsT, 11 mapped to genes or operons expressed differently in response to perturbation of vpsT (Table 1) (20). Consistent with expectation, most VpsT peaks locate to non-coding DNA upstream of a gene 5' end (Figure 1c). The function of each gene flanking a binding peak is illustrated in Figure 1d. Many encode proteins implicated in biofilm formation, c-di-GMP metabolism, or motility. We used electrophoretic mobility shift assays (EMSAs) to test direct binding of VpsT with 8 of the ChIP-seq derived targets (Figure S2a). Binding was confirmed in all cases. Conversely, VpsT did not bind the Escherichia coli lacZ promoter, which was used as a negative control (Figure S2b).

\section{Reassessment of the T-box consensus sequence for VpsT binding}

The precise sequence of the T-box, the DNA motif recognized by VpsT, is not clear. In initial DNAse I footprinting studies, Zamorano-Sánchez et al. showed binding of VpsT to a DNA palindrome at the $v p s L$ regulatory region (30). A near identical sequence was identified at the vpsA, $v p s T, r b m A$ and $r b m E$ promoters (30). Direct binding of VpsT has also been demonstrated at the rpoS locus using DNAse I footprinting (28). However, the bound sequence differed to the motif previously found (28). Other direct VpsT targets also lack the palindrome identified by ZamoranoSánchez et al. (26, 27, 29). Logically, our ChIP-seq analysis should provide a starting point for better understanding of the T-box consensus. To identify motifs common to all ChIP-seq targets we used MEME to parse DNA sequences centred on each of the $23 \mathrm{VpsT}$ binding peaks. The motif identified is shown in Figure 1e (top) alongside the palindrome previously described by ZamoranoSánchez et al. (bottom) (30). To aid comparison, we numbered positions in both palindromes with respect to the centre of the two sequences (Figure 1e). Whilst the ChIP-seq derived logo is shorter, and exhibits greater degeneracy, there are similarities between the sequences. Most notably, 
positions $-3 \mathrm{C}$ and $+3 \mathrm{G}$ of both motifs are highly conserved and likely to be important for VpsT binding.

\section{Mutagenesis of the vpsL promoter T-box}

Recall that Zamorano-Sánchez et al. used DNAse I footprinting to locate VpsT binding upstream of $v p s L$ (30). Figure 2a illustrates the $v p s L$ intergenic region, numbered with respect to the primary TSS (30). The centre of the VpsT ChIP-seq peak is marked by an asterisk. Positions in bold align to the motif found using ChIP-seq. Overlapping sequences matching the original motif of Zamorano-Sánchez and colleagues are underlined (30). To further understand the requirements for VpsT binding we made a series of point mutations. The base changes, numbered with respect to the vpsL TSS, are marked red in Figure 2a. To measure the impact of the mutations we used DNAse I footprinting (Figure 2b). As expected, VpsT bound the vpsL promoter region poorly in the absence of c-di-GMP (compare lanes 1 and 2). Conversely, in the presence of c-di-GMP, VpsT could elicit a substantial footprint (lane 3). Mutations -236C (lanes 4 and 5) -233T (lanes 6 and 7) and -220G (lanes 12 and 13) target positions in the Zamorano-Sánchez et al. logo but beyond the boundaries of the ChIP-seq derived motif. None of these mutations altered VpsT binding. Mutations -228T and -223T target positions conserved in both motifs. Whilst the -228T mutation had no impact (lanes 8 and 9) the -223T mutation abolished VpsT binding (lanes 10 and 11).

\section{Comparison of sequences protected from DNAse I digestion by VpsT}

As noted above, prior studies pinpointed VpsT binding, at targets other than vpsL, by DNAse I footprinting $(28,29)$. For comparison, we used the same approach to locate binding of VpsT at the aer and $f r h C$ regulatory regions identified by ChIP-seq (Table 1). At the aer locus, VpsT produced two distinct regions of DNA protection that overlapped the centre of the ChIP-seq binding signal (Figure S3). A similar pattern of binding was observed upstream of $f r h C$, except that three sections of DNA were bound by VpsT (Figure S4). None of the sequences protected by VpsT, here or in past work, match the T-box sequence identified by Zamorano-Sánchez et al (30). We reasoned that the T-box motif identified by ChIP-seq might allow us to resolve these apparent discrepancies. Hence, we collated all eight sequences bound by VpsT in footprinting experiments. We then searched for the ChIP-seq derived T-box motif within these sequences (Figure 3c). In seven cases, an obvious match to the motif was found. In one instance ( $r$ rhC T-box I) a motif was found but the 
most highly conserved positions, usually $-3 \mathrm{C}$ and $+3 \mathrm{G}$, were inverted (Figure 2c, Figure S4b). Note that the T-box sequence motif suggests that this may occasionally be the case (Figure 1e). We conclude that the ChIP-seq derived logo, generated with the benefit of more VpsT target site information, better describes the DNA binding properties of VpsT.

\section{VpsT binds two T-boxes upstream of the vibrio phase variation operon}

Definition of the T-box consensus should permit easier dissection of gene regulation by VpsT. Hence, we focused our attention on the vibrio phase variation ( $v p v)$ operon that contains three genes: $v p v A, v p v B$ and $v p v C$. Importantly, whilst VpvA and $\mathrm{VpvB}$ are hypothetical proteins of unknown function, $\mathrm{VpvC}$ is a diguanylate cyclase contributing to the corrugated morphology of rugose phase variants (31). The ChIP-seq data for VpsT binding at the $v p v A B C$ locus is shown in Figure 3a. The sequence of the intergenic region is shown in Figure $3 \mathrm{~b}$ where the centre of the ChIP-seq peak is indicated by an asterisk. Note that sequences are numbered according to distance from the 3' end of the DNA sequence. To precisely identify the location of VpsT binding we again used DNAse I footprinting (Figure 3c). In the absence of c-di-GMP no VpsT binding was detected (lanes 1-5). However, in the presence of c-di-GMP, two sections of the regulatory region were protected from DNAse I digestion (lanes 6-10). The regions of protection, each $\sim 20 \mathrm{bp}$ in length, overlap the centre of the ChIP-seq peak and are indicated by cyan boxed sequences in Figure $3 b$. Examination of the sequences identified T-box motifs (bold in Figure 3b) that we named T-box I and T-box II. Note that T-box I has the same sequence inversion at positions -3 and +3 described above for frhC T-box I.

\section{The regulatory region of the vibrio phase variation operon contains two promoters}

To begin understanding the regulatory role of VpsT we searched for TSSs, upstream of $v p v A B C$, using primer extension assays. To do this, the DNA sequence shown in Figure $3 \mathrm{~b}$ was cloned upstream of lacZ in plasmid pRW50T. The resulting DNA construct was transferred into $V$. cholerae E7946 by conjugation. These cells were then transformed with pAMNF, or pAMNF-vpsT, so that we could determine the consequence of VpsT expression. Finally, RNA was extracted and an oligonucleotide complementary to $l a c Z$ was used to prime reverse transcription. An electrophoretic analysis of the resulting cDNA products is shown in Figure $3 \mathrm{~d}$. In the absence of ectopic VpsT expression, we could detect a single cDNA (Figure 3d, lane 1). This primer 
extension product indicates a TSS located at position 151 of the cloned $v p v A B C$ regulatory region (marked orange in Figure 3b). In the presence of VpsT, we could detect an additional cDNA species (Figure 3d, lane 2). The second extension product results from a TSS located at position 61 of the cloned $v p v A B C$ regulatory region (marked purple in Figure $3 b$ ). We named the promoters associated with each TSS $v p v A \mathrm{P} 1$ and $v p v A \mathrm{P} 2$, according to their proximity to the $v p v A$ start codon (Figure 3b). We note that Papenfort and co-workers previously used dRNA-seq to map TSSs genome-wide in $V$. cholerae (32). This analysis, done using conditions not expected to induce VpsT controlled genes, identified only the $v p v A \mathrm{P} 2 \mathrm{TSS}$. We conclude that $v p v \mathrm{P} 1$ is a strictly VpsT activated promoter. The constitutive activity of $v p v A \mathrm{P} 2$ is likely a result of better matches to promoter element consensus sequences (Figure 3b).

\section{Activation of vpvAPl by VpsT requires the adjacent T-box}

To further understand interplay between the promoter and T-box sequences we made derivatives of the $v p v A B C$ regulatory region. The DNA fragments are illustrated schematically in Figure 4a and the different 5 ' ends are marked by inverted triangles in Figure $3 \mathrm{~b}$. The various sequences were fused to lacZ in plasmid pRW50T and $\beta$-galactosidase activity was determined in lysates of $V$. cholerae strain E7946, with or without ectopic VpsT expression. As expected, the starting regulatory fragment was able to stimulate lac $Z$ expression and $\beta$-galactosidase activity doubled when VpsT was produced (Figure 4b, wild type). To understand the contribution of vpvAP2 promoter, and the overlapping T-box II, we removed 60 base pairs from the 5 ' end of the wild type DNA fragment. The truncated DNA sequence, named $\triangle v p v A \mathrm{P} 2$, stimulated lower levels of $\beta$-galactosidase activity but the fold induction by VpsT was unaltered (Figure 4b, $\Delta v p v A \mathrm{P} 2$ ). Hence, activation of $v p v A \mathrm{P} 1$ by VpsT does not require T-box II. To confirm the importance of T-box I we altered the sequence of this DNA element in the context of the truncated DNA fragment. The mutations are indicated by red text in Figure $3 \mathrm{~b}$ and the resulting DNA fragment was named $\triangle v p v A \mathrm{P} 2^{\mathrm{Mut}}$. Mutation of T-box I abolished activation of $v p v A \mathrm{P} 1$ by VpsT (Figure 4b, $\left.\Delta v p v A \mathrm{P} 2^{\mathrm{Mut}}\right)$. In a final experiment, we deleted T-box I completely and activation by VpsT was similarly ablated (Figure $4 \mathrm{~b}, \Delta v p v A \mathrm{P} 2^{\Delta \mathrm{T}}$ ). 


\section{The vpvAP1 promoter and T-box I are needed to maintain intracellular c-di-GMP levels}

Previous work has shown that diguanylate cyclase activity generates c-di-GMP (13) and that this molecule positively regulates the activity of VpsT (20). In turn, we show that VpsT activates expression of $v p v A B C$, which encodes a diguanylate cyclase. We reasoned that this should constitute a positive feedback loop dependent on T-box I. To test this, we made alterations to the chromosomal $v p v A B C$ regulatory region. Specifically, we deleted a 60 bp section of chromosomal DNA, containing $v p v A \mathrm{P} 2$ and T-box II, before making a derivative of this strain with point mutations in T-box I. The chromosomal changes are equivalent to the $\Delta v p v A \mathrm{P} 2$ and $\triangle v p v A \mathrm{P} 2^{\mathrm{Mut}}$ constructs described above for lacZ fusion assays. We also examined the effect of deleting vpsT or $v p v A B C$. To assess the consequences, we used a plasmid encoded intracellular c-di-GMP biosensor (33). Briefly, the system relies on expression of two different fluorescent reporters. One reporter, AmCyan, is produced constitutively and serves as a normalisation control. The second reporter, TurboRFP, is regulated by a c-di-GMP activated riboswitch. Intracellular c-di-GMP levels are inferred by calculating the AmCyan:TurboRFP ratio. These ratios are shown in Figure 5 alongside images of representative colonies. Wild type colonies have an orange appearance due to the combined expression of AmCyan and TurboRFP. Cells lacking vpsT formed colonies with a green-blue appearance due to greatly reduced c-di-GMP levels. Surprisingly, complete deletion of the $v p v A B C$ operon had no effect. Similarly, deletion of $v p v A \mathrm{P} 2$ did not induce a large change in c-di-GMP levels. Conversely, mutation of the $v p v A B C$ T-box I, required for activation of $v p v A \mathrm{P} 1$ by VpsT, reduced intracellular levels of c-di-GMP by $40 \%$.

\section{The vpvAP2 promoter is important for $V$. cholerae phase variation}

In a final set of experiments, we wanted to understand the contribution of the different $v p v A B C$ operon regulatory elements to phase variation. Smooth to rugose phase variation in $V$. cholerae can be induced by growing cells in nutrient depleted media (34). We inoculated alkaline phosphate water using a single smooth colony of each strain. After incubation, cells were plated and the number of colonies with the smooth or rugose phenotype were counted. The results are shown in Table 2. As expected, deletion of vpsT significantly reduced smooth to rugose phase variation by 4-fold. Conversely, complete deletion of $v p v A B C$ had no effect. Deletion of $v p v A P 2$ caused a significant 2-fold increase in rugose colony numbers that did not change significantly upon deletion of T-box I needed to activate the remaining $v p v A \mathrm{P} 1$ promoter. We conclude that $v p v A \mathrm{P} 2$ impacts phase variation but $v p v A \mathrm{P} 1$ does not. 


\section{CONCLUSIONS}

We argue that the T-box is best described as a degenerate palindrome with the consensus 5 '- ${ }_{-5} \mathrm{R}$. ${ }_{4}[\mathrm{CG}]_{-3} \mathrm{Y}_{-2} \mathrm{~W}_{-1} \mathrm{~W}_{+1} \mathrm{R}_{+2}[\mathrm{GC}]_{+3} \mathrm{Y}_{+4} \mathrm{~W}_{+5}-3$ '. This resembles part of a prior T-box motif, generated from a smaller number of targets, and explains observations of VpsT binding diverse sequences (Figure 2c) (28-30). Positions -3 and +3 of the motif are key and, whilst usually $\mathrm{C}$ and $\mathrm{G}$ respectively, exhibit the complimentary sequence in some cases (Figures $2 \mathrm{c}$ and $3 \mathrm{~b}$ ). In both scenarios, mutation of the key positions prevents interaction with VpsT (Figures $2 \mathrm{~b}$ and 4b). Globally, the DNA binding properties of VpsT are intriguing. Despite recognising a highly degenerate sequence, VpsT targets just 23 loci in our ChIP-seq assay (Table 1). Furthermore, whilst vps $T$ resides within $V$. cholerae chromosome II, all VpsT targets locate to chromosome I. This is largely consistent with prior transcriptome analysis, which identified a similar skew in VpsT regulated genes (20). The unusual distribution is unlikely to be driven solely by occurrence of the T-box sequence; incidence of the motif can be identified frequently across both chromosomes (35). Hence, the ability of VpsT to bind DNA must be influenced by additional factors. Other regulators and DNA supercoiling could both play a role. We note that VpsT appears to bind DNA differently at certain targets. For instance, at the vpsL regulatory region, DNA protection stretched $\sim 60$ base pairs upstream of the T-box (Figure 2). This protection cannot be due to VpsT independently binding multiple sites; a single T-box point mutation was sufficient to abolish the footprint. Hence, we suggest that VpsT must cooperatively oligomerise across, or tightly wrap, the vpsL regulatory DNA. The VpsT binding pattern is different upstream of $v p v A B C$; a precise footprint of each T-box is observed. Interestingly, VpsT activates vpsL transcription by antagonising H-NS mediated repression (25, 30). This is not the case for $v p v A B C$, that does not bind H-NS (25). Hence, we speculate that the more extensive interactions of $\mathrm{VpsT}$ at the $v p s L$ locus may be necessary to displace H-NS.

Our data suggest that the $v p v A \mathrm{P} 1$ and $v p v A \mathrm{P} 2$ promoters have different roles. The latter is constitutively active whilst $v p v A P 1$ is activated by VpsT (Figure 3) (32). The constitutive activity of $v p v A \mathrm{P} 2$ is consistent this promoter impacting the rate of phase variation; rugose colonies can result from mutation of $v p v C$, basal expression of which requires $v p v A \mathrm{P} 2$ (Figure 3d) $(31,32)$. Conversely, the VpsT activated vpvAP1 promoter did not impact phase variation (Table 2). However, this promoter did impinge on c-di-GMP homeostasis. This is likely a result of a positive 
feedback loop; c-di-GMP activates VpsT that subsequently upregulates expression of VpvC and so diguanylate cyclase activity. Hence, mutation of T-box I, needed to activate $v p v A \mathrm{P} 1$, led to a 40 $\%$ decrease in intracellular c-di-GMP (Figure 5). Deletion of vpsT caused the intracellular c-di-GMP signal to decrease dramatically (Figure 5). However, this phenotype may depend on regulatory targets in addition to $v p v A B C$. The stability of the intracellular c-di-GMP signal following $v p v A B C$ deletion suggests other DGC proteins play a compensatory role. Indeed, $V$. cholerae encode thirty one proteins with the "GGDEF" motif and redundancy is common $(15,36)$. Furthermore, as the roles of $v p v A$ and $v p v B$ are less well defined, their deletion could have confounding consequences. In conclusion, VpsT both relays the c-di-GMP signal and is important for the control of intracellular c-di-GMP levels.

\section{MATERIALS AND METHODS}

\section{Strains, plasmids and oligonucleotides}

Strains, plasmids and oligonucleotides used in this study are listed in Table S1. All V. cholerae strains are derivatives of E7946 (37). Chromosomal deletions and mutants were made using either the pKAS32 suicide plasmid for allelic exchange or the MuGENT method (38, 39). The Escherichia coli strain JCB387 was used for routine cloning (40). Plasmids were transferred into V. cholerae by either conjugation or transformation as described previously $(41,42)$.

\section{ChIP-seq and bioinformatics}

The chromatin immunoprecipitation assay was done as in prior work (42) using strain E7946, carrying plasmid pAMNF-vpsT, or empty pAMNF as a control. Note that pAMNF-vpsT encodes 3xFLAG-VpsT that is expressed from a low level constitutively active promoter (43). The VpsTDNA complexes were immunoprecipitated with an anti-FLAG antibody (Sigma) and Protein A sepharose beads. Immunoprecipitated DNA was blunt-ended, A-tailed, and ligated to barcoded adaptors before elution and de-crosslinking. ChIP-seq libraries were then amplified by PCR and purified. Library quality was assessed using an Agilent Tapestation 4200 instrument and quantity determined by qPCR using an NEBnext library quantification kit (NEB). Libraries were sequenced as described previously (43) and reads are available from ArrayExpress using accession code EMTAB-10829. Single-end reads from two independent VpsT ChIP-seq experiments were mapped to the reference $V$. cholerae N16961 genome (chromosome I: NC_002505.1 and chromosome II: NC_002506.1) with Bowtie 2 using the QuasR package. MACS2 was used to call ChIP-seq peaks, 
which were then visually inspected using the Artemis genome browser to confirm peak centres $(44,45)$. Average read counts were normalised according to the coverage per base for each experiment and visualised using Gviz.

\section{$\beta$-galactosidase activity assays}

Promoter DNA was fused to lacZ in plasmid pRW50T that can be transferred from $E$. coli to $V$. cholerae by conjugation (41). Assays of $\beta$-galactosidase activity were done according to the Miller method (46). Bacterial cultures were grown in LB broth, supplemented with appropriate antibiotics, to mid-log phase. Values shown are the mean of three independent experiments and error bars show the standard deviation.

\section{Electrophoretic mobility shift assays and DNAse I footprinting}

We purified VpsT as described previously (29). Promoter DNA fragments were excised from plasmid pSR and end-labelled with $\gamma 32$-ATP using T4 PNK (NEB). EMSAs and DNase I footprints were done as previously described (42). Full gel images are shown in Figure S5.

\section{Primer extension assays}

The RNA for primer extension assays was purified from $V$. cholerae carrying appropriate plasmids as described above. Primer extension assays were done as previously described (47). The 5' endlabelled primer D49724, which anneals downstream of the HindIII site in pRW50T, was used in all experiments. Primer extension products were analysed on denaturing 6\% polyacrylamide gels, calibrated with arbitrary size standards, and visualized using a Fuji phosphor screen and Bio-Rad Molecular Imager FX. Full gel images are shown in Figure S5.

\section{Colony fluorescence quantification}

Cultures of $V$. cholerae were grown aerobically overnight in LB. A $2 \mu \mathrm{L}$ suspension was spotted onto LB agar plates that were incubated overnight at $30^{\circ} \mathrm{C}$. The fluorescence signal from each of the two fluorophores was measured with a ChemiDoc MP system (Bio-Rad) as previously described (33). Image Lab (BioRad) was used to measure the fluorescence intensity, with the same number of pixels recorded for each colony. The ratio of TurboRFP to the AmCyan signal was then calculated. Values shown are mean ratios from 4 independent experiments, as a proportion of the wild type ratio. 


\section{ACKNOWLEDGEMENTS}

This work was funded by BBSRC project grant BB/N005961/1 awarded to DCG and a BBSRC MIBTP studentship (BB/M01116X/1, project reference 1790836) awarded to TG. We thank Fitnat Yildiz for the gift of plasmid pFY4535 and critical reading of the manuscript prior to submission.

\section{FIGURE LEGENDS}

Figure 1: Genome-wide distribution of VpsT in Vibrio cholerae. a) Binding of VpsT across both Vibrio cholerae chromosomes. The outer two tracks (navy blue) depict genes in the forward and reverse orientation. The VpsT ChIP-seq signal (cyan) is shown by the inner track. b) ChIPseq coverage plots for known VpsT targets. In each case the VpsT ChIP-seq signal shown in cyan is the average of reads aligned from two independent experiments. Block arrows in navy blue indicate genes that are labelled by name and/or locus tag. Those genes known to be regulated by VpsT are in bold type face. c) Position of VpsT binding peaks with respect to genes. The distribution of VpsT binding peaks occurring at 100 bp intervals from the nearest coding gene. Inset pie chart shows the proportion of peaks that occur in coding and non-coding DNA. d) Pie chart showing the predicted function of genes adjacent to VpsT ChIP-seq peaks. e) DNA sequence logos representing the VpsT binding sequence, or T-box, motif. The upper logo is derived from all 23 VpsT ChIP-seq peaks and the lower sequence was previously reported by Zamorano-Sánchez et al (30).

Figure 2: Mutagenesis of the vpsL regulatory region T-box. a) DNA sequence of the regulatory region upstream of $v p s L$. The T-box proposed by Zamorano-Sánchez et al. is underlined (30). The bold typeface identifies the shorter T-box consensus consistent with all ChIP-seq targets. Mutations made to the T-box are shown below in red. The primary vpsL transcription start site (TSS) is indicated by a bent arrow. Regulatory region positions are labelled with respect to the primary vpsL TSS (+1). The cyan box highlights the section of the regulatory region protected from DNAse I digestion by VpsT. b) Image of a denaturing polyacrylamide gel used to analyse DNAse I digestion of the vpsL regulatory region. The gel is calibrated with a Maxam-Gilbert ' $\mathrm{G}+\mathrm{A}$ ' ladder. The presence and absence of VpsT $(6 \mu \mathrm{M})$ and c-di-GMP $(50 \mu \mathrm{M})$ is indicated. c) Alignment of DNA sequences protected by VpsT in DNAse I footprinting experiments. Other than for the $\operatorname{rpoS}$ and $V C 1303 / V C 1304$ loci $(28,29)$, the footprinting data are from the present study. 
Sequences are aligned according to the position of the T-box (bold type). Aside from vpsL, where the sequence was too long, the full protected top strand sequence is shown in the 5 ' to $3^{\prime}$ direction. The ChIP-seq derived T-box logo is shown above the aligned sequences for comparison. In the alignment, positions coloured red, green, orange or blue match either the preferred or second most common base according to the ChIP-seq derived motif. Sequences in bold black type match the third or fourth most frequent base.

Figure 3: Binding of VpsT upstream of the $v p v A B C$ operon activates transcription. a) Binding of VpsT to the $v p v A B C$ regulatory region in vivo. The VpsT ChIP-seq signal shown in cyan is the average of reads aligned from two independent experiments. Block arrows in navy blue indicate genes that are labelled by name and/or locus tag. The solid black bar indicates the location of the DNA sequence shown in panel b. b) DNA sequence of regulatory region upstream of $v p v A B C$. Bold typeface indicates the T-box consensus identified by ChIP-seq. The asterisk indicates the centre of the ChIP-seq peak for VpsT binding. The cyan box highlights the section of the regulatory region protected from DNAse I digestion by VpsT. Mutations made to the T-box are shown in red. Transcription start sites (+1) are indicated by bent arrows. The -10 and -35 elements of the associated $v p v A \mathrm{P} 1$ and $v p v A \mathrm{P} 2$ promoters are also highlighted and matches to the consensus are underlined. Regulatory region positions are labelled with respect to the 3 ' end of the DNA fragment used in subsequent DNAse I footprinting experiments. The $v p v A$ start codon is coloured navy blue. Inverted triangles indicate sites of truncation to generate derivatives of the regulatory DNA. c) Picture of a denaturing polyacrylamide gel used to separate DNA fragments resulting from DNAse I digestion of the $v p v A B C$ regulatory region. The gel is calibrated with a Maxam-Gilbert ' $\mathrm{G}+\mathrm{A}$ ' ladder. The presence and absence of $\operatorname{VpsT}(2,4,6$ or $8 \mu \mathrm{M})$ and c-di-GMP $(50 \mu \mathrm{M})$ is indicated. d) Identification of transcription start sites upstream of $v p v \mathrm{ABC}$. The gel image shows the cDNA products of a primer extension assay. Arbitrary Maxam-Gilbert ' $\mathrm{G}+\mathrm{A}$ ' ladders were used to calibrate the gel. The RNA used for primer extension was extracted from V. cholerae strain E7946 carrying the $v p v A B C$ regulatory region cloned in plasmid pRW50T. The cells also harboured empty plasmid pAMNF (- VpsT, lane 1) or pAMNF-vpsT (+ VpsT, lane 2). Transcription start sites are labelled according to the schematic in panel $b$. 
Figure 4: Activation of the vpvAP1 promoter requires VpsT and the adjacent T-box. a) Schematic representation of $v p v A B C$ regulatory region DNA fragments fused to lacZ. Truncations are at the sites indicated in Figure $3 \mathrm{~b}$ and remove T-box II and/or $v p v A \mathrm{P} 2$ promoter. Transcription start sites are shown by bent arrows and labelled according to the name of the associated promoter. The wild type or mutated T-box I is shown cyan or red respectively. The downstream lacZ gene is represented by a block arrow. b) Measurements of $\beta$-galactosidase activity in the presence and absence of VpsT. Activities were measured for derivatives of $V$. cholerae strain E7946 encoding the different regulatory region fragments cloned in the $l a c Z$ reporter plasmid pRW50T. The cells also carried empty plasmid pAMNF (- VpsT) or pAMNF-vpsT (+ VpsT). Error bars show standard deviation for three independent biological replicates.

\section{Figure 5: Activation of $v p v A B C$ by VpsT is needed to maintain intracellular c-di-GMP levels} in Vibrio cholerae. Relative intracellular concentrations of c-di-GMP were determined in V. cholerae strain E7946 and derivatives using a c-di-GMP biosensor (33). The system uses expression of two different fluorescent reporters; constitutive AmCyan expression serves as a normalisation control and TurboRFP expression requires c-di-GMP. Relative intracellular c-diGMP levels are determined by calculating the TurboRFP:AmCyan ratio for different strains. The two lower panels show representative colonies imaged using blue epi illumination and a 530/28 filter (for AmCyan fluorescence) or green epi illumination and a 605/50 filter (for TurboRFP). A photograph taken in natural light is shown for comparison. The top panel shows a bar chart comparing TurboRFP:AmCyan ratio for different strains. The bars are coloured to match the TurboRFP signal in the bottom image. The strains are distinguished by chromosomal deletions and mutations indicated below the figure. The chromosomal changes at the $v p v A B C$ regulatory region are equivalent to those described in Figure 4a for promoter::lacZ fusions. Values shown are the average of 4 independent experiments and error bars indicate standard deviation.

\section{Figure 6: Model describing the different roles of VpsT.}

Schematic showing the proposed interaction between VpsT, VpvC and c-di-GMP. 


\section{SUPPLEMENTARY FIGURE LEGENDS}

Figure S1: Comparison of VpsT levels in different strains. The figure shows the result of a western blotting experiment, with anti-FLAG antibodies, to detect chromosomal or plasmid encoded 3xFLAG-VpsT. Each measurement was done in triplicate for the strains indicated above the gel image.

Figure S2: Binding of purified VpsT in vitro to DNA targets identified by ChIP-seq in vivo. a) The images show results from electrophoretic mobility shift assays with purified VpsT $(0-7.5 \mu \mathrm{M})$, c-di-GMP $(50 \mu \mathrm{M})$ and DNA fragments corresponding to ChIP-seq peaks detected for VpsT binding in vivo. b) A negative control electrophoretic mobility shift assay demonstrating no binding of VpsT to Escherichia coli lacZ DNA.

Figure S3: VpsT binds two targets at the aer regulatory region. a) Binding of VpsT to the aer regulatory region in vivo. The VpsT ChIP-seq signal shown in cyan is the average of reads aligned from two independent experiments. Block arrows in navy blue indicate genes that are labelled by name and/or locus tag. The solid black bar indicates the location of the DNA sequence shown in panel b. b) DNA sequence of regulatory region upstream of aer. Bold typeface indicates the T-box consensus identified by ChIP-seq. The asterisk indicates the centre of the ChIP-seq peak for VpsT binding. The cyan box highlights the section of the regulatory region protected from DNAse I digestion by VpsT. c) Image of a denaturing polyacrylamide gel used to separate DNA fragments resulting from DNAse I digestion of the aer regulatory region. The gel is calibrated with a MaxamGilbert ' $\mathrm{G}+\mathrm{A}$ ' ladder. The presence and absence of $\operatorname{VpsT}(2,4,6$ or $8 \mu \mathrm{M})$ and c-di-GMP $(50 \mu \mathrm{M})$ is indicated.

Figure S4: VpsT binds three targets at the $f r h A / f r h C$ regulatory region. a) Binding of VpsT to the frhA/frhC regulatory region in vivo. The VpsT ChIP-seq signal shown in cyan is the average of reads aligned from two independent experiments. Block arrows in navy blue indicate genes that are labelled by name and/or locus tag. The solid black bar indicates the location of the DNA sequence shown in panel b. b) DNA sequence of regulatory region upstream of $f r h C$. Bold typeface indicates the T-box consensus identified by ChIP-seq. The asterisk indicates the centre of the ChIPseq peak for VpsT binding. The cyan box highlights the section of the regulatory region protected 
from DNAse I digestion by VpsT. The frhC start codon is in blue text. c) Image of a two denaturing polyacrylamide gels used to separate DNA fragments resulting from DNAse I digestion of the DNA sequence upstream of $f r h C$. The length of the regulatory DNA meant that it was necessary to label the $5^{\prime}$ ends of either the (i) bottom or (ii) top DNA strands to get full coverage of the sequence. The gel is calibrated with a Maxam-Gilbert ' $\mathrm{G}+\mathrm{A}$ ' ladder. The presence and absence of VpsT $(2,4,6$ or $8 \mu \mathrm{M})$ and c-di-GMP $(50 \mu \mathrm{M})$ is indicated.

c) Image of a denaturing polyacrylamide gel used to separate DNA fragments resulting from DNAse I digestion of the aer regulatory region. The gel is calibrated with a Maxam-Gilbert ' $\mathrm{G}+\mathrm{A}$ ' ladder. The presence and absence of $\operatorname{VpsT}(2,4,6$ or $8 \mu \mathrm{M})$ and c-di-GMP $(50 \mu \mathrm{M})$ is indicated.

Figure S5: Original gel images. Original uncropped gel images used for this work. Coloured boxes indicate the approximate boundaries of images following cropping of images for figures. 


\section{REFERENCES}

1. Charles,R.C. and Ryan,E.T. (2011) Cholera in the 21st century. Curr. Opin. Infect. Dis., 24, $472-477$.

2. Mutreja,A., Kim,D.W., Thomson,N.R., Connor,T.R., Lee,J.H., Kariuki,S., Croucher,N.J., Choi,S.Y., Harris,S.R., Lebens,M., et al. (2011) Evidence for several waves of global transmission in the seventh cholera pandemic. 477, 462-465.

3. Domman,D., Quilici,M.-L., Dorman,M.J., Njamkepo,E., Mutreja,A., Mather,A.E., Delgado,G., Morales-Espinosa,R., Grimont,P.A.D., Lizárraga-Partida,M.L., et al. (2017) Integrated view of Vibrio cholerae in the Americas. Science, 358, 789-793.

4. Weill,F.-X., Domman,D., Njamkepo,E., Tarr,C., Rauzier,J., Fawal,N., Keddy,K.H., Salje,H., Moore,S., Mukhopadhyay,A.K., et al. (2017) Genomic history of the seventh pandemic of cholera in Africa. Science, 358, 785-789.

5. Nelson,E.J., Harris,J.B., Morris,J.G., Calderwood,S.B. and Camilli,A. (2009) Cholera transmission: the host, pathogen and bacteriophage dynamic. Nat. Rev. Microbiol., 7, 693702.

6. Rawlings,T.K., Ruiz,G.M. and Colwell,R.R. (2007) Association of Vibrio cholerae O1 El Tor and $\mathrm{O} 139$ Bengal with the copepods Acartia tonsa and Eurytemora affinis. Appl. Environ. Microbiol., 73, 7926-7933.

7. Meiborn,K.L., Li,X.B., Nielsen,A.T., Wu,C.Y., Roseman,S. and Schoolnik,G.K. (2004) The Vibrio cholerae chitin utilization program. PNAS, 101, 2524-2529.

8. Berk,V., Fong,J.C.N., Dempsey,G.T., Develioglu,O.N., Zhuang,X., Liphardt,J., Yildiz,F.H. and Chu,S. (2012) Molecular architecture and assembly principles of Vibrio cholerae biofilms. Science, 337, 236-239.

9. Fong,J.C.N. and Yildiz,F.H. (2007) The rbmBCDEF gene cluster modulates development of rugose colony morphology and biofilm formation in Vibrio cholerae. J. Bacteriol., 189, 2319-2330.

10. Yildiz,F.H. and Schoolnik,G.K. (1999) Vibrio cholerae O1 El Tor: Identification of a gene cluster required for the rugose colony type, exopolysaccharide production, chlorine resistance, and biofilm formation. PNAS, 96, 4028-4033.

11. Matz,C., McDougald,D., Moreno,A.M., Yung,P.Y., Yildiz,F.H. and Kjelleberg,S. (2005) Biofilm formation and phenotypic variation enhance predation-driven persistence of Vibrio cholerae. PNAS, 102, 16819-16824.

12. Fong,J.C.N., Karplus,K., Schoolnik,G.K. and Yildiz,F.H. (2006) Identification and Characterization of RbmA, a Novel Protein Required for the Development of Rugose Colony Morphology and Biofilm Structure in Vibrio cholerae. J. Bacteriol., 188, 10491059.

13. Hengge,R. (2009) Principles of c-di-GMP signalling in bacteria. Nat. Rev. Microbiol. 2009 
74, 7, 263-273.

14. Ausmees,N., Mayer,R., Weinhouse,H., Volman,G., Amikam,D., Benziman,M. and Lindberg,M. (2001) Genetic data indicate that proteins containing the GGDEF domain possess diguanylate cyclase activity. FEMS Microbiol. Lett., 204, 163-167.

15. Conner,J.G., Zamorano-Sánchez,D., Park,J.H., Sondermann,H. and Yildiz,F.H. (2017) The ins and outs of cyclic di-GMP signaling in Vibrio cholerae. Curr. Opin. Microbiol., 36, 20.

16. Pursley,B.R., Maiden,M.M., Hsieh,M.L., Fernandez,N.L., Severin,G.B. and Waters,C.M. (2018) Cyclic di-GMP regulates TfoY in Vibrio cholerae to control motility by both transcriptional and posttranscriptional mechanisms. J. Bacteriol., 200.

17. BR,P., NL,F., GB,S. and CM,W. (2019) The Vc2 Cyclic di-GMP-Dependent Riboswitch of Vibrio cholerae Regulates Expression of an Upstream Putative Small RNA by Controlling RNA Stability. J. Bacteriol., 201.

18. Kariisa,A.T., Weeks,K. and Tamayo,R. (2016) The RNA Domain Vc1 Regulates Downstream Gene Expression in Response to Cyclic Diguanylate in Vibrio cholerae. PLoS One, 11, e0148478.

19. Rege,M., Subramanian,V., Zhu,C., Hsieh,T.H.S., Weiner,A., Friedman,N., ClauderMünster,S., Steinmetz,L.M., Rando,O.J., Boyer,L.A., et al. (2015) Chromatin Dynamics and the RNA Exosome Function in Concert to Regulate Transcriptional Homeostasis. Cell Rep., 13, 1610-1622.

20. Krasteva,P. V., Jiunn,J.C., Shikuma,N.J., Beyhan,S., Navarro,M.V.A.S., Yildiz,F.H. and Sondermann,H. (2010) Vibrio cholerae vpst regulates matrix production and motility by directly sensing cyclic di-GMP. Science, 327, 866-868.

21. Teschler,J.K., Zamorano-Sánchez,D., Utada,A.S., Warner,C.J.A., Wong,G.C.L., Linington,R.G. and Yildiz,F.H. (2015) Living in the matrix: Assembly and control of Vibrio cholerae biofilms. Nat. Rev. Microbiol., 13, 255-268.

22. Casper-Lindley,C. and Yildiz,F.H. (2004) VpsT Is A Transcriptional Regulator Required for Expression of vps Biosynthesis Genes and the Development of Rugose Colonial Morphology in Vibrio cholerae O1 El Tor. J. Bacteriol., 186, 1574-1578.

23. FH,Y., NA,D. and GK,S. (2001) VpsR, a Member of the Response Regulators of the TwoComponent Regulatory Systems, Is Required for Expression of vps Biosynthesis Genes and EPS(ETr)-Associated Phenotypes in Vibrio cholerae O1 El Tor. J. Bacteriol., 183, 17161726.

24. Fong,J.C.N., Syed,K.A., Klose,K.E. and Yildiz,F.H. (2010) Role of Vibrio polysaccharide (vps) genes in VPS production, biofilm formation and Vibrio cholerae pathogenesis. Microbiology, 156, 2757-2769.

25. Ayala,J.C., Wang,H., Silva,A.J. and Benitez,J.A. (2015) Repression by H-NS of genes required for the biosynthesis of the Vibrio cholerae biofilm matrix is modulated by the second messenger cyclic diguanylic acid. Mol. Microbiol., 97, 630-645. 
26. Fernandez,N.L., Srivastava,D., Ngouajio,A.L. and Waters,C.M. (2018) Cyclic di-GMP positively regulates DNA repair in Vibrio cholerae. J. Bacteriol., 200.

27. Fernandez,N.L. and Waters,C.M. (2019) Cyclic di-GMP increases catalase production and hydrogen peroxide tolerance in Vibrio cholerae. Appl. Environ. Microbiol., 85.

28. Wang,H., Ayala,J.C., Benitez,J.A. and Silva,A.J. (2014) The LuxR-type regulator VpsT negatively controls the transcription of rpos, encoding the general stress response regulator, in Vibrio cholerae biofilms. J. Bacteriol., 196, 1020-1030.

29. Warman,E.A., Forrest,D., Guest,T., Haycocks,J.J.R.J., Wade,J.T. and Grainger,D.C. (2021) Widespread divergent transcription from bacterial and archaeal promoters is a consequence of DNA-sequence symmetry. Nat. Microbiol., 6, 746-756.

30. Zamorano-Sánchez,D., Fong,J.C.N.N., Kilic,S., Erill,I. and Yildiz,F.H. (2015) Identification and Characterization of VpsR and VpsT Binding Sites in Vibrio cholerae. J. Bacteriol., 197, $1221-1235$.

31. Beyhan,S. and Yildiz,F.H. (2007) Smooth to rugose phase variation in Vibrio cholerae can be mediated by a single nucleotide change that targets c-di-GMP signalling pathway. Mol. Microbiol., 63, 995-1007.

32. Papenfort,K., Förstner,K.U., Cong,J.P., Sharma,C.M. and Bassler,B.L. (2015) Differential RNA-seq of Vibrio cholerae identifies the VqmR small RNA as a regulator of biofilm formation. PNAS, 112, E766-E775.

33. Zamorano-Sánchez,D., Xian,W., Lee,C.K., Salinas,M., Thongsomboon,W., Cegelski,L., Wong,G.C.L. and Yildiz,F.H. (2019) Functional Specialization in Vibrio cholerae Diguanylate Cyclases: Distinct Modes of Motility Suppression and c-di-GMP Production. MBio, 10.

34. Ali,A., Rashid,M.H. and Karaolis,D.K.R. (2002) High-Frequency Rugose Exopolysaccharide Production by Vibrio cholerae. Appl. Environ. Microbiol., 68, 5773.

35. Guest,T. (2021) Understanding the Role of VpsT in Vibrio cholerae.

36. Massie,J.P., Reynolds,E.L., Koestler,B.J., Cong,J.-P., Agostoni,M. and Waters,C.M. (2012) Quantification of high-specificity cyclic diguanylate signaling. PNAS, 109, 12746-12751.

37. Levine,M.M., Black,R.E., Clements,M.L., Cisneros,L., Saah,A., Nalin,D.R., Gill,D.M., Craig,J.P., Young,C.R. and Ristaino,P. (1982) The pathogenicity of nonenterotoxigenic vibrio cholerae serogroup 01 biotype ei tor isolated from sewage water in brazil. J. Infect. Dis., 145, 296-299.

38. K,S. and RK,T. (1996) Positive selection vectors for allelic exchange. Gene, 169, 47-52.

39. Dalia,A.B., McDonough,E.K. and Camilli,A. (2014) Multiplex genome editing by natural transformation. PNAS, 111, 8937-8942.

40. Page,L., Griffiths,L. and Cole,J.A. (1990) Different physiological roles of two independent pathways for nitrite reduction to ammonia by enteric bacteria. Arch. Microbiol., 154, 349- 
354.

41. Manneh-Roussel,J., Haycocks,J.R.J., Magán,A., Perez-Soto,N., Voelz,K., Camilli,A., Krachler,A.M. and Grainger,D.C. (2018) Camp receptor protein controls Vibrio cholerae gene expression in response to host colonization. MBio, 9.

42. Haycocks,J.R.J.J., Warren,G.Z.L.L., Walker,L.M., Chlebek,J.L., Dalia,T.N., Dalia,A.B. and Grainger,D.C. (2019) The quorum sensing transcription factor AphA directly regulates natural competence in Vibrio cholerae. PLoS Genet., 15, e1008362.

43. Sharma,P., Haycocks,J.R.J., Middlemiss,A.D., Kettles,R.A., Sellars,L.E., Ricci,V., Piddock,L.J. V and Grainger,D.C. (2017) The multiple antibiotic resistance operon of enteric bacteria controls DNA repair and outer membrane integrity. Nat. Commun., 8, 1444.

44. Carver,T., Harris,S.R., Berriman,M., Parkhill,J. and Mcquillan,J.A. (2012) Artemis: an integrated platform for visualization and analysis of high-throughput sequence-based experimental data. 28, 464-469.

45. Zhang,Y., Liu,T., Meyer,C.A., Eeckhoute,J., Johnson,D.S., Bernstein,B.E., Nusbaum,C., Myers,R.M., Brown,M., Li,W., et al. (2008) Model-based Analysis of ChIP-Seq (MACS). Genome Biol. 2008 99, 9, 1-9.

46. Miller,J. (1972) Experiments in Molecular Genetics.

47. Haycocks,J.R.J. and Grainger,D.C. (2016) Unusually situated binding sites for bacterial transcription factors can have hidden functionality. PLoS One, 11.

48. Haycocks,J.R.J., Sharma,P., Stringer,A.M., Wade,J.T. and Grainger,D.C. (2015) The Molecular Basis for Control of ETEC Enterotoxin Expression in Response to Environment and Host. PLoS Pathog., 11, 1-16.

49. Bolivar,F., Rodriguez,R.L., Betlach,M.C. and Boyer,H.W. (1977) Construction and characterization of new cloning vehicles. I. Ampicillin-resistant derivatives of the plasmid pMB9. Gene, 2, 75-93.

50. Kolb,A., Kotlarz,D., Kusano,S. and Ishihama,A. (1995) Selectivity of the Escherichia coli RNA polymerase E $\sigma 38$ for overlapping promoters and ability to support CRP activation. Nucleic Acids Res., 23, 819-826.

51. Ditta,G., Stanfield,S., Corbin,D., Helinski,D.R. and Donald Helinskl,C.R. (1980) Broad host range DNA cloning system for Gram-negative bacteria: Construction of a gene bank of Rhizobium meliloti (plasmid RK2/plasmid vehicle/conjugal transfer/nif genes). Genet. Downloaded Univ. Birmingham, 77, 7347-735. 
Table 1: VpsT binding peaks identified by ChIP-seq

\begin{tabular}{llcl} 
Peak centre $^{1}$ & Adjacent gene(s) $^{\mathbf{2}}$ & Bound by H-NS $^{\mathbf{3}}$ & Known VpsT target $^{\mathbf{4}}$ \\
\hline 383248 & VC0364 & no & no \\
540341 & VC0510<>VC0512 & no & no \\
544169 & VC0513 & no & no \\
564171 & VC0534 & no & yes* \\
888647 & VC0825<>VC0826 & yes & no \\
891494 & VC0829 & no & no \\
903390 & VC0841 & yes & no \\
979211 & VC0917 & yes & yes* \\
990915 & VC0928 & yes & yes* \\
999012 & VC0932<>VC0934 & yes & yes* \\
1096963 & VC1029<>VC1031 & no & yes \\
1385301 & VC1303<>VC1304 & no & no \\
1412875 & VC1329 & yes & no \\
1741639 & VC1620<>VC1621 & no & yes \\
1845393 & VC1710<>VC1711 & no & yes \\
1947982 & VC1802<>VC1803 & no & no \\
1990005 & VC1851 & no & no \\
2221277 & VC2065 & no & yes \\
2336167 & VC2188 & no & yes \\
2376037 & VC2221 & no & no \\
2640531 & VC2456 & no & yes \\
2835743 & VC2667 & no & yes
\end{tabular}

${ }^{1}$ All co-ordinates refer to $V$. cholerae N16961 chromosome I.

${ }^{2}$ Genes listed are located closest to the ChIP-seq peak. Peaks between divergent genes are marked "<>".

${ }^{3}$ Overlapping H-NS binding in ChIP-seq experiments (25).

${ }^{4}$ According to gene expression profiling (20). Cases where direct binding of VpsT has been shown are denoted by an asterisk. 
Table 2: Conversion of smooth to rugose variants in different genetic backgrounds

\begin{tabular}{|c|c|c|c|c|}
\hline \multirow[b]{2}{*}{ Strain ${ }^{1}$} & \multicolumn{2}{|c|}{ Number of colonies ${ }^{2}$} & \multirow[b]{2}{*}{$\%$ Rugose $^{3}$} & \multirow[b]{2}{*}{$p$ value $^{4}$} \\
\hline & Smooth & Rugose & & \\
\hline Wild type & 142 & 85 & 37 & N/A \\
\hline$\Delta v p s T$ & 271 & 24 & 8 & $<0.00001$ \\
\hline$\triangle v p v A B C$ & 60 & 42 & 41 & 0.520214 \\
\hline$\triangle v p v A \mathrm{P} 2$ & 28 & 57 & 67 & $<0.00001$ \\
\hline$\Delta v p v A \mathrm{P} 2^{\mathrm{Mut}}$ & 36 & 45 & 56 & 0.127989 \\
\hline
\end{tabular}

${ }^{1}$ V. cholerae strain E7946 or derivatives thereof.

${ }^{2} \mathrm{~A}$ single smooth colony was used to inoculate alkaline phosphate water. After 72 hours incubation, cells were plated and the number of colonies with the smooth or rugose phenotype were counted.

${ }^{3}$ The percentage of all colonies counted that had a rugose phenotype.

${ }^{4}$ All $p$ values were calculated using the Chi-square test. We compared the number of smooth and rugose colonies for the wild type parent strain with each derivative. The exception was the $\triangle v p v A \mathrm{P} 2^{\mathrm{Mut}}$ strain that was compared with $\triangle v p v A \mathrm{P} 2$. The null hypothesis was that no differences between strains would be observed. 
Table S1: Strains, plasmids and oligonucleotides

Name

\section{Escherichia coli strains}

DH5 $\alpha$ endA1, glnV44, thi-1, recA1, relA1, gyrA96, deoR, nupG, purB20, NEB $\varphi 80 \mathrm{~d} l a c Z \Delta \mathrm{M} 15, \Delta(\mathrm{lacZYA}-\arg F) \mathrm{U169}, h s d R 17(\mathrm{rK}-\mathrm{mK}+), \lambda$

JCB387 $\Delta$ nirB, $\Delta l a c$

S17 $\lambda$ pir $\quad$ lacU169 (lacZM15), recA1, endA1, hsdR17, thi-1, gyrA96, relA1, pir

Vibrio cholerae strains

E7946 Wildtype Vibrio cholerae El Tor Ogawa derivative, SmR

E7946 E7946 derivative lacking vpsT

This work

$\Delta v p s T$

E7946 E7946 derivative lacking $v p v A B C$

This work

$\triangle v p v A B C$

E7946

E7946 derivative with a 60 bp deletion that removes the vpvAP2

This work

$\triangle v p v A \mathrm{P} 2$

promoter and most of the overlapping T-box I

E7946 E7946 $v p v A \mathrm{P} 2$ derivative with two point mutations in T-box II

This work $\triangle v p v A \mathrm{P} 2^{\mathrm{Mut}}$

\section{Plasmids}

pET28a High copy number plasmid for expression of N-terminal His6-tagged Novagen proteins, KanR

pAMNF Plasmid for basal expression of N-terminal 3xFLAG-tagged proteins, (48) KanR

pRW50T A broad-host range lacZ expression vector encoding oriT of pRK,

TetR, Tra+

pBR322 General cloning vector, AmpR, TetR

pSR $\quad 4 \mathrm{~kb}$ pBR322 derivative that encodes AmpR. Contains an EcoRI-

HindIII cloning site upstream of the $\lambda$ oop transcription terminator 
pRK2013 Helper plasmid for conjugation, KanR, oriColE1, RK2-, Mob+, Tra+ (51)

pKAS32 Suicide plasmid for mutant strain construction, AmpR

pFY4535 pFY4357 containing the hok/sok region from $\mathrm{pXB} 300, \mathrm{GmR}$

Oligonucleotides for promoter cloning and mutagenesis $\left(5^{\prime} \text { to } 3^{\prime}\right)^{1}$

VC0510|1.1F GGCTGCGAATTCCATTTACATTTGATTGGTATAATAATTCGC

VC0510|1.1R GCCCGAAGCTTCATTTCGGGCTCCTGTCGGAATCAATGG

VC0512|1.1R GCCCGAAGCTTCATTTCGGGCTCCTGTCGGAATCAATGG

VC0512|1.2F GGCTGCGAATTCCATTTCGGGCTCCTGTCGGAATCAATGG

VC1030|1.1F GGCTGCGAATTCTGACTTTCAGTGAGTCCATCCTGTTCTCC

VC1030|1.1R GCCCGAAGCTTCACCGACCTGATTCCTAATGAGTGATCGC

VC1031|1.1F GGCTGCGAATTCCACTGTCCGTACCTCCCCATGCTTCGATAT ACACCCC

VC1031|1.1R GCCCGAAGCTTCATCTCACGCTGATTGAGTTAAGTGTTGT TC AAAATGAGCC

VC1621|1.1F GGCTGCGAATTCCATATAAAGCCTCAGACCCTAATAC

VC1621|1.1R GCCCGAAGCTTCATTACTCAATGAATGACCTTTAGTC

VC1620|1.1R GCCCGAAGCTTCATATAAAGCCTCAGACCCTAATAC

VC1620|1.1F GGCTGCGAATTCCATTACTCAATGAATGACCTTTAGTC

VC1710|1.1R GCCCGAAGCTTCATCCGGTATCCCTAAACTGATGATG

VC1710|1.1F GGCTGCGAATTCCATATTTTGGCCTCAAGATTAAGAGGGC

VC1711|1.1F GGCTGCGAATTCCATCCGGTATCCCTAAACTGATGATG

VC1711|1.1R GCCCGAAGCTTCATATTTTGGCCTCAAGATTAAGAGGGC

VC1851|1.1R GCCCGAAGCTTCAAAGATTTACCTATTTTCGGGTTGTTG

VC1851|1.1F GGCTGCGAATTCACACCGCCCTGCAATTCACTCCATTC

VC2188|1.2F GGCTGCGAATTCCAGTTAGTTGAAGAATTTCATACCGCCTTT ATCCATCTC

VC2188|1.1R GCCCGAAGCTTCATAGTTTGCTCTCCTATCGAGTTCGC

VC2456|1.1F GGCTGCGAATTCTGCGGCGTTCATGCTTTGGTTCATTTC

VC2456|1.1R GCCCGAAGCTTCAAGTGAAATATTCTCCATTTCGATCGTC

$\triangle v p v A P 2 \mid \mathrm{F} \quad$ GGCTGCGAATTCGTGCTTAATTTTCC

$\Delta v p v A P 2^{\Delta \mathrm{T}} \mid \mathrm{F}$ GGCTGCGAATTCCTTTGCACCCAACG

$\triangle v p v A P 2|\mathrm{R}|$ GGGTGCAAAGAGTCATAAAATTCATTCTTCCGTTGG

G120|C115|

vpsL|wt|1.1|F GGCTGCGAATTCGTTCTGTTTTTCCTTTC

$v p s L|w t| 1.1 \mid \mathrm{R}$ GCCCGAAGCTTACTAGACGCTCCTAACC

vpsL|-233T|R TGAAAATAAACTTTAGTTTAATTTTATGATGGTTAATAGG

vpsL|-223T|R ATTAACCACCATGAAAATAAÄATTAGTTTACTTTTATGATGG

vpsL|-236C TAACCTATTAACCATCATACĀ̈GTAAACTAAAGTTTATTT

vpsL|-228T TTAACCATCATAAAAGTAĀTTAAAGTTTATTTTCATGGTG

vpsL|-220G ATAAAAGTAAACTAAAGTTGĀTTTCATGGTGGTTAATAA

This work

This work

This work

This work

This work

This work

This work

This work

This work

This work

This work

This work

This work

This work

This work

This work

This work

This work

This work

This work

This work

This work

This work

This work

This work

This work

This work

This work

This work

This work

This work

This work 
Oligonucleotides for cloning N-terminal FLAG-tagged VpsT in pAMNF (5' to 3')

$\begin{array}{llr}\text { VpsT|VC| } & \text { GGCTGCGGTACCAAAGAtGAAAACAAACtAAACGTTAGAAT } & \text { This work } \\ \text { Nter|For } & \text { GCTTTCTG } & \text { This work } \\ \text { VpsT|VC| } & \text { GCCCGAAGCTTTTAAGAATtGACTTCCTCAATTCCAATATT } & \\ \text { Nter|Rev } & \text { ATtTTTCGC } & \end{array}$

Oligonucleotides cloning VpsT in pET28a (5' to 3')
VpsT|Nhis|F cCCAATCCATATGAAAgAtgAAAACAAACTAAACGTTAGA This work ATGCTTTC
VpsT|Nhis|R GgCGGATCCTtAAGAATtgACTTCCTCAATTCCAATATTAT $\quad$ This work TTTTCGC

Oligonucleotides to construct an N-terminal $3 x F L A G$ chromosomal vpsT (5' to $\left.3^{\prime}\right)$

$\begin{array}{lll}\text { up|flank|fwd } & \text { TAGAGGTACCGGTTGTTAACtACTAAACGAAATTACCGTAT } & \text { This work } \\ \text { up|flank|rev } & \text { C TGGAATAAACATTTCACCCCTCCTAAC } & \text { This work } \\ \text { down|flank| } & \text { CCAAGGGTACCAAAGATGAAAACAAACTAAACG } & \text { This work } \\ \text { fwd } & & \\ \text { down|flank| } & \text { CGCCAGCTGCAGGCGGCCGCTAACATTTTTAAAGATCTGTT } & \text { This work } \\ \text { rev } & \text { TGC } & \\ \text { flag|fwd } & \text { GGGTGAAATGATTTATTCCAATGTCACACAC } & \text { This work } \\ \text { flag|rev } & \text { TTTCATCTTTGGTACCCTTGTCATCGTC } & \text { This work }\end{array}$

Oligonucleotides used for MuGENT to delete vpsT (5' to 3')

gw|Mugent| ACTCGAAGAGACATTACACAACGAATTCACCCCTCCTAACA $\quad$ This work vpsT|F2| CATCAAGGC

$\mathrm{RC}+\mathrm{R} 1$

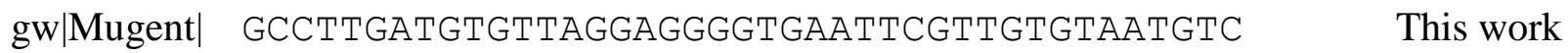
vpsT|R1| TCTTCGAGT

$\mathrm{RC}+\mathrm{F} 2$

Oligonucleotides for construction of mutations at the chromosomal vpvABC locus (5' to 3')

pKAS32|fwd GCGGCCGCCTGCAGCTGG

pKAS32|rev GTTAACAACCGGTACCTCTAGAACTATAGCTAGCATGCGC vpvA|p2| TTAGTGATTGGTGAGACAAAAATACCCGTTGGGTGCAAAG deletion|m1| AGTCATAAAAT

exo|F1.1

pkAS32|vpv|f ATGGATGGTGATGCCATCCGGCGGCCGCCTGCAGCTGGCG pkAS32|vpv|r AGTTGAGCAGTACATAGTCGCTCTGTACATGTCCGCGGTC
This work This work This work

This work This work 


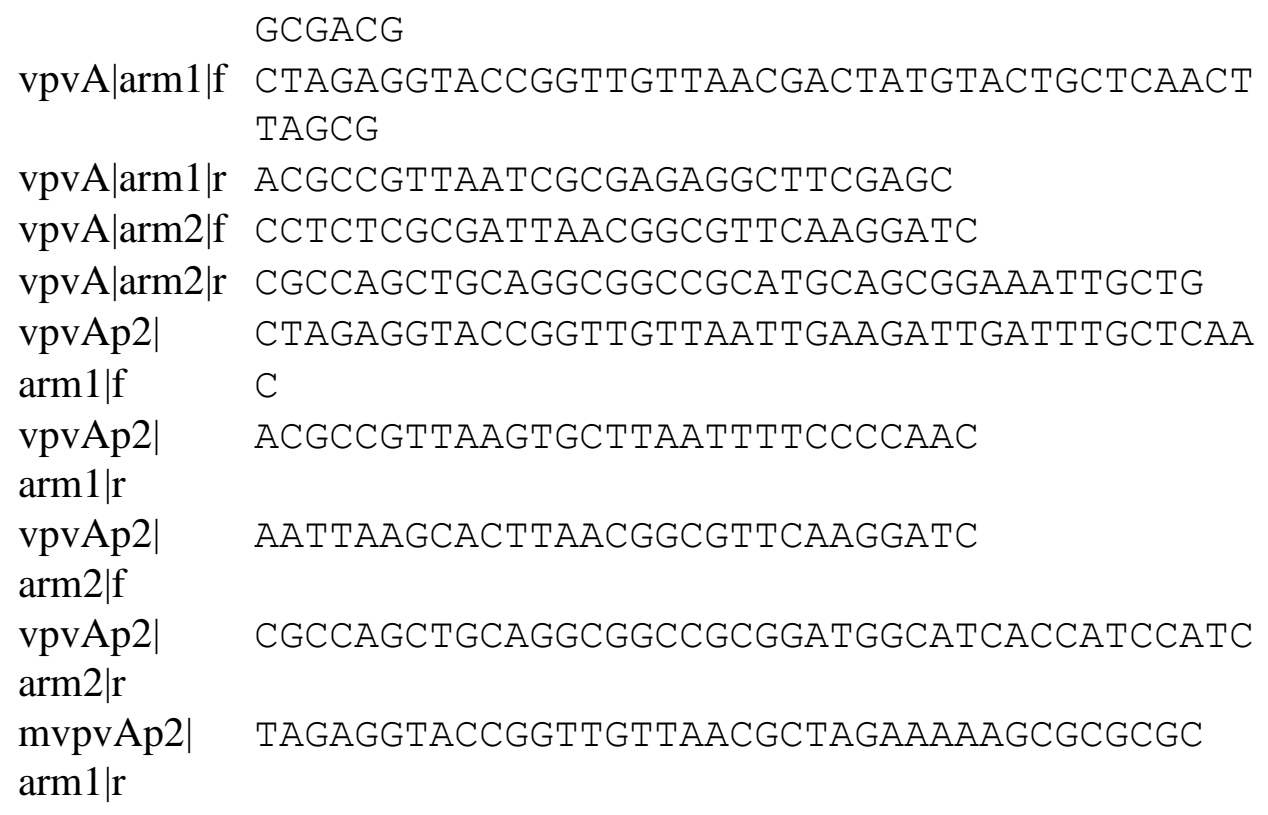

${ }^{1}$ positions of point mutations are underlined

This work

This work This work This work This work This work

This work This work This work 
a available under aCC-BY 4.0 International license.

Figure 1
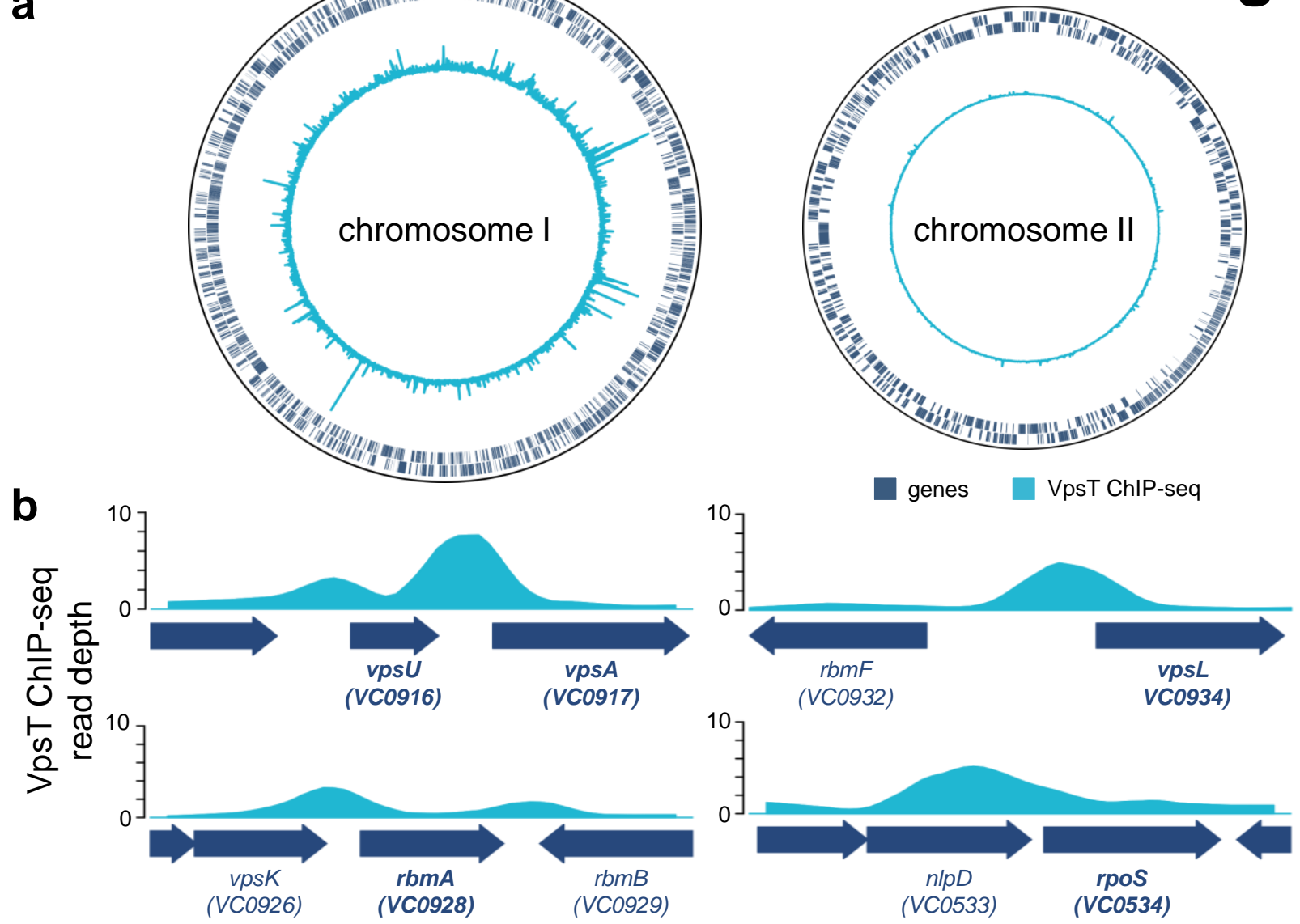

C

Position of VpsT binding peaks with respect to gene start codons

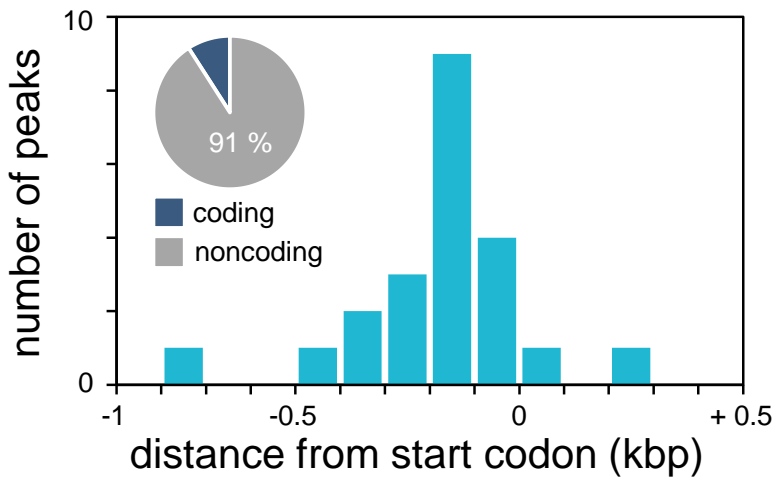

d

Functions encoded by genes adjacent to VpsT binding peaks

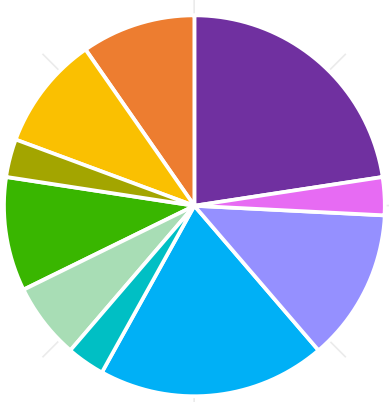

Biofilm formation c-di-GMP metabolism Cell adhesion

Chemotaxis

Metabolism Motility Other

Secretion system

Transcriptional regulator Unknown

e

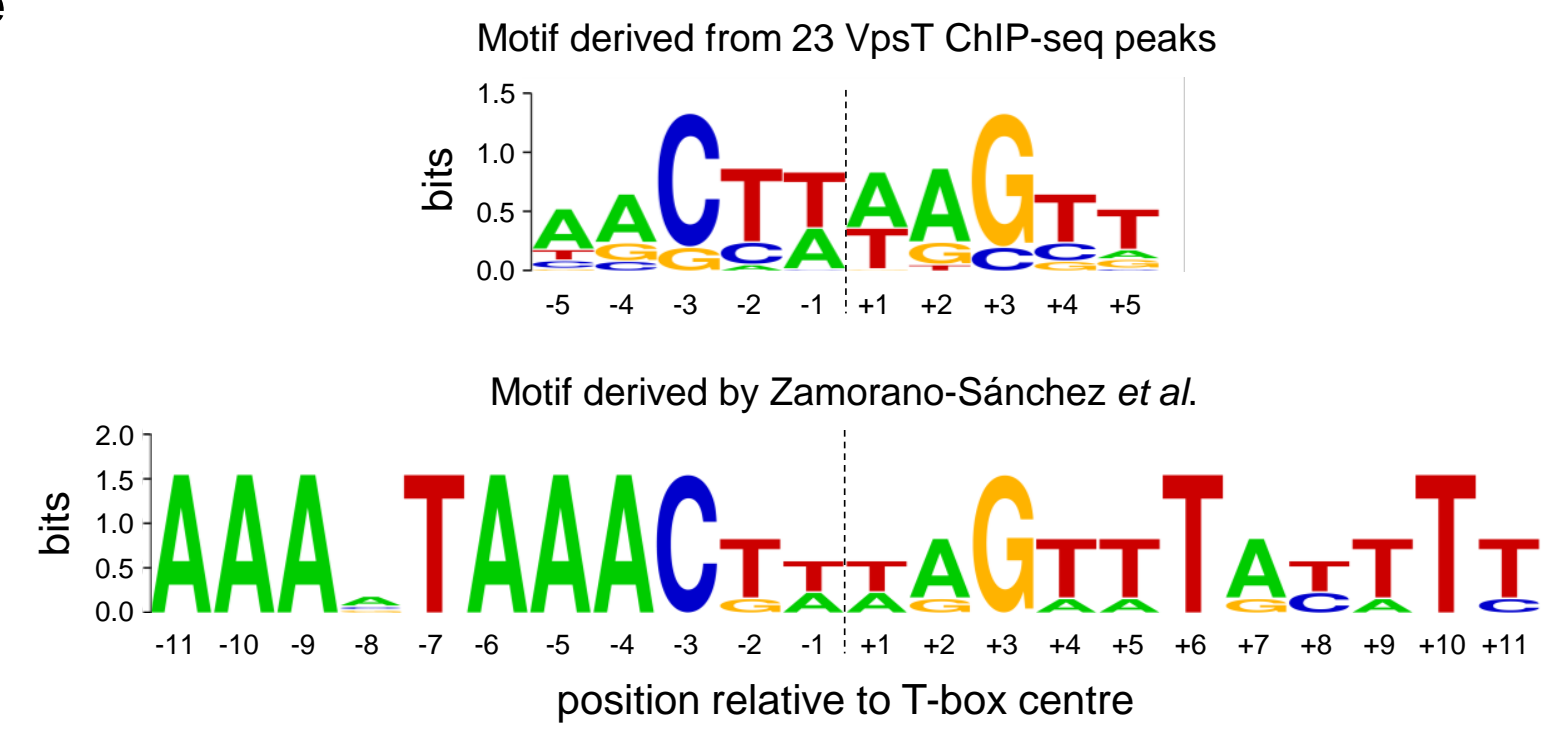


a

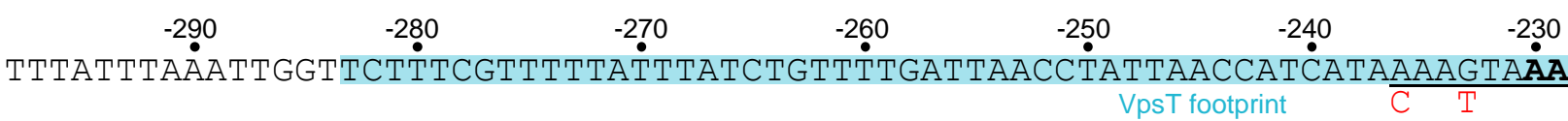

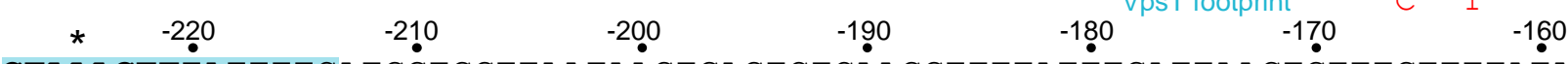
CTAAAGTTTATTTTCATGGTGGTTAATA $\dot{T}$ GTGAGTCTCÄAGGTTTTATTTGATTAAGTǴTTTGTTTTATA

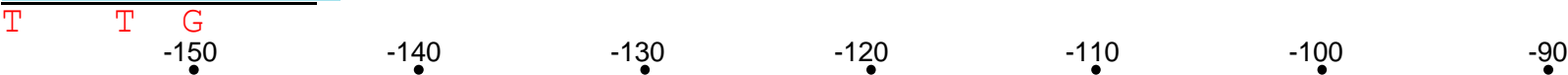

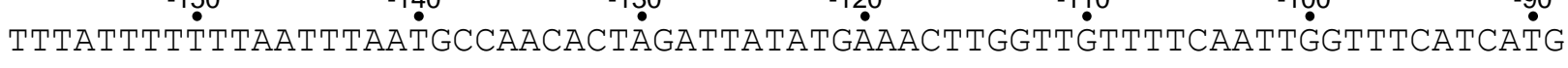
$\begin{array}{rrrrrr}-80 & -70 & -60 & -50 & -40 & -30\end{array}$

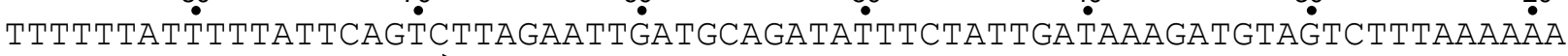
GAATTTAAं̈ACATTCCAATT

b

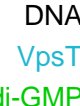

㐫
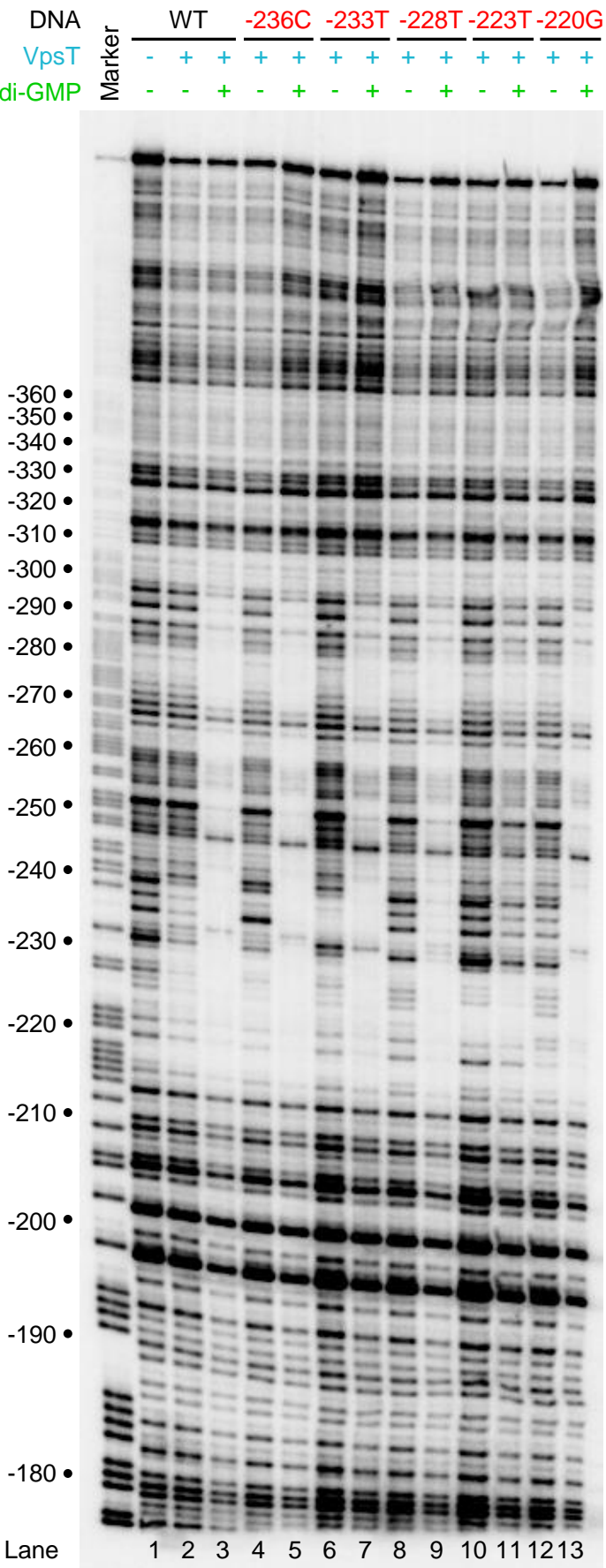

\section{C}

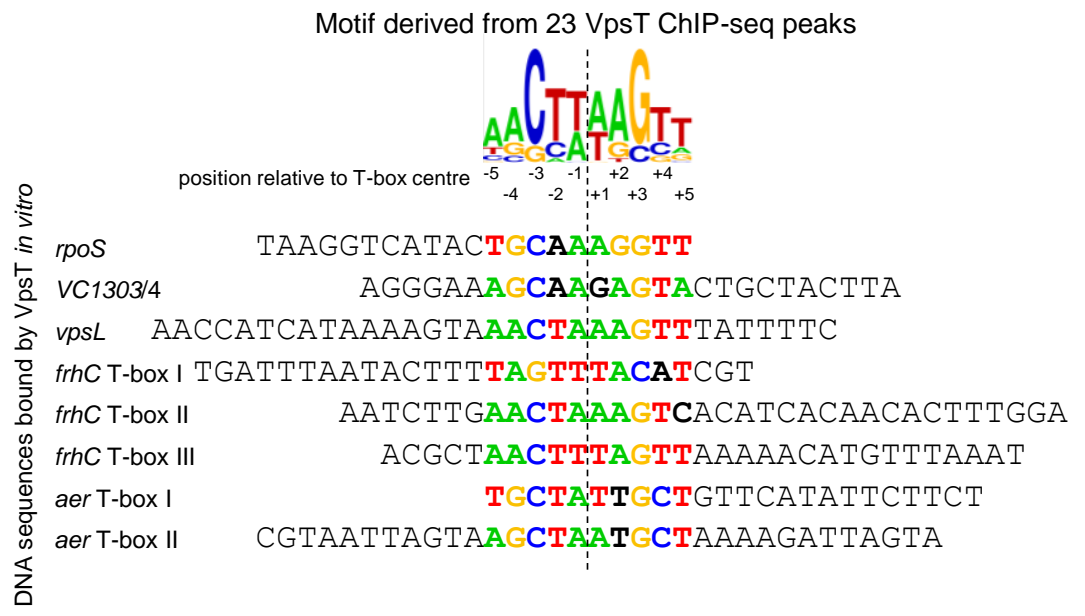

Motif derived from 23 VpsT ChIP-seq peaks

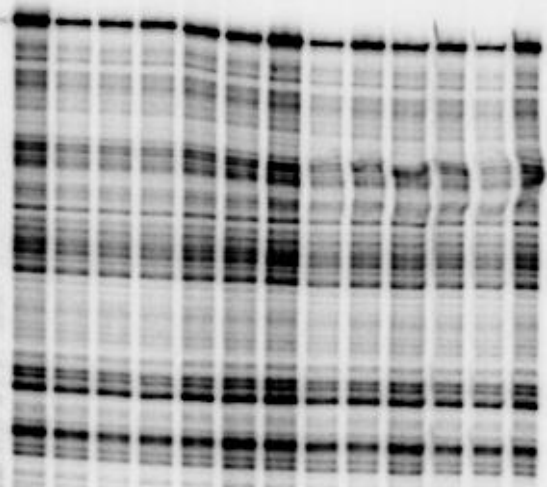

\section{TAAGGTCATACTGCAA'AGGTT}

AGGGAAAGCAAGAGTACTGCTACTTA

a vpl AACCATCATAAAAGTAAACTA'AAGTTTATTTTC

frhC T-box I TGATTTAATACTTTTAGTTMACATCGT

frhCT-box II

frhC T-box II

क्ष aer T-box

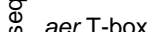

合
ACGCTAACTTTAGTTAAAAACATGTTTAAAT
TGCTATT TCTGTTCATATTCTTCT

CGTAATTAGTAAGCTA'A TACTAAAAGATTAGTA 


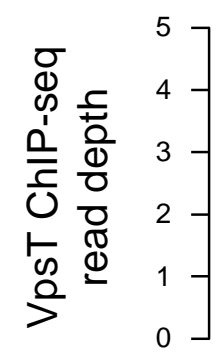

Genes

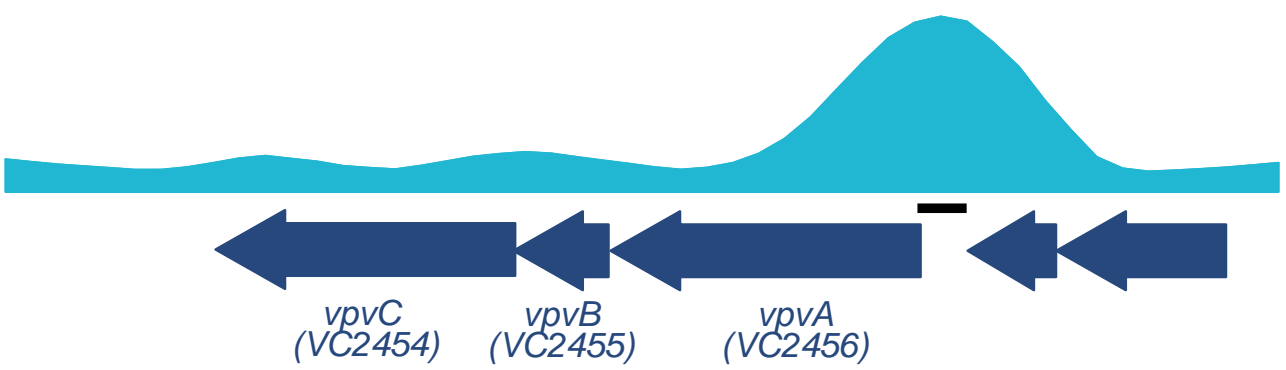

b

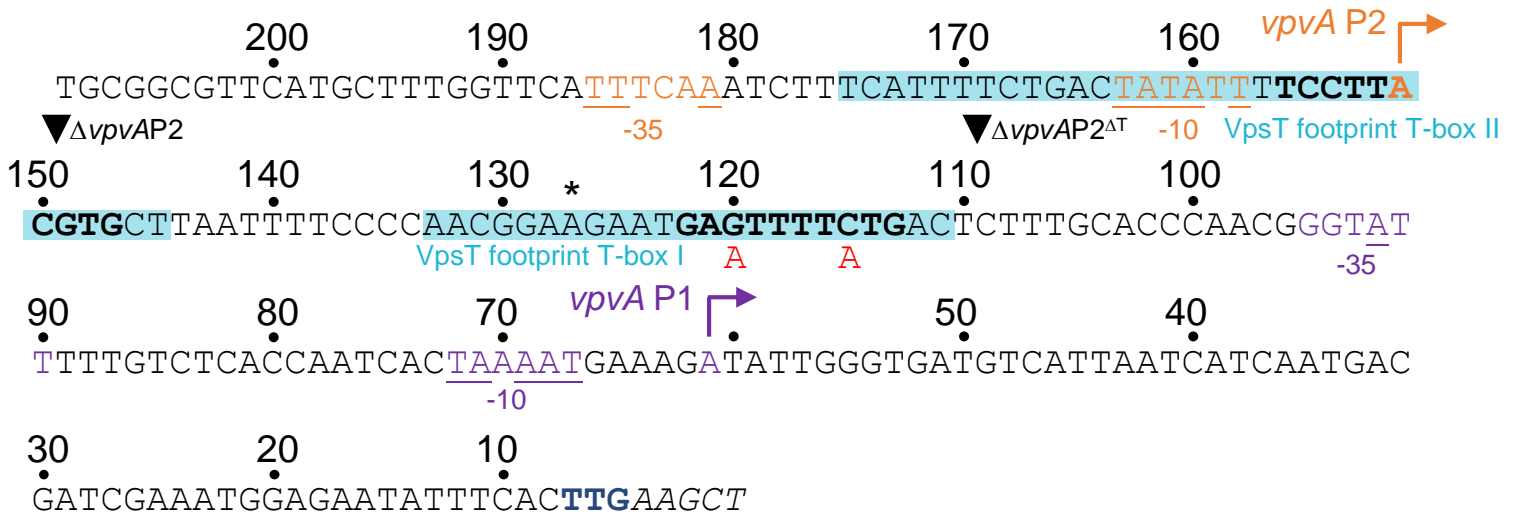

C
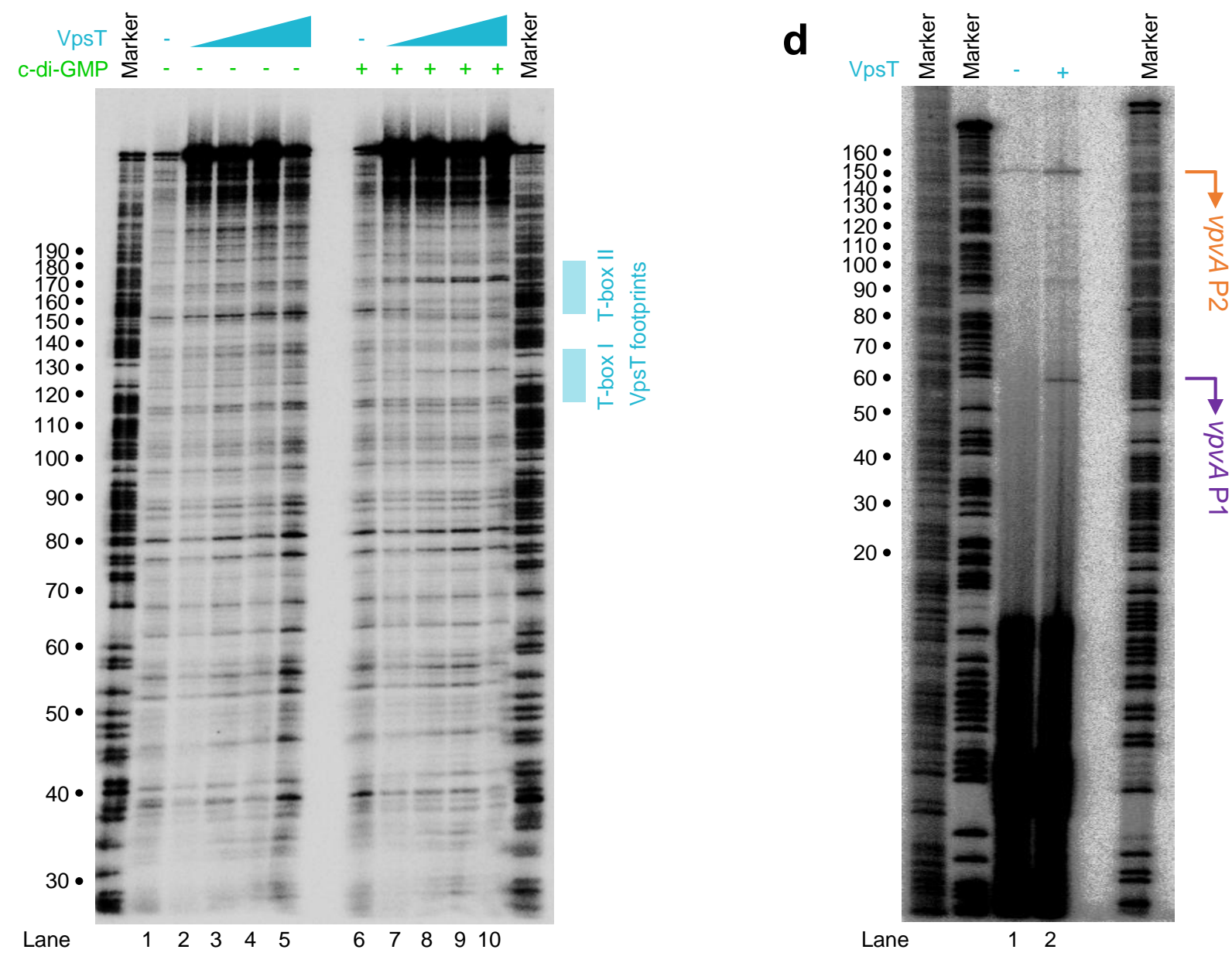
a

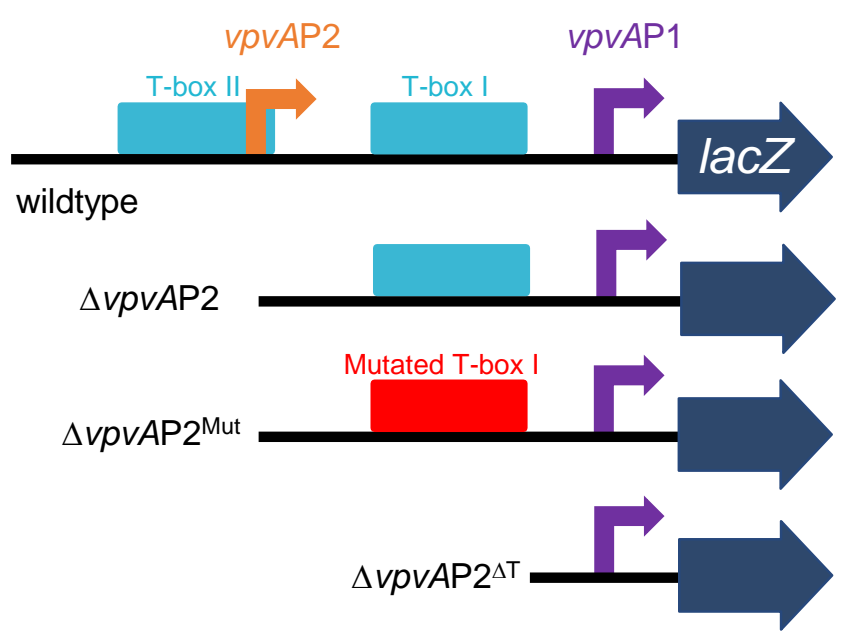

b

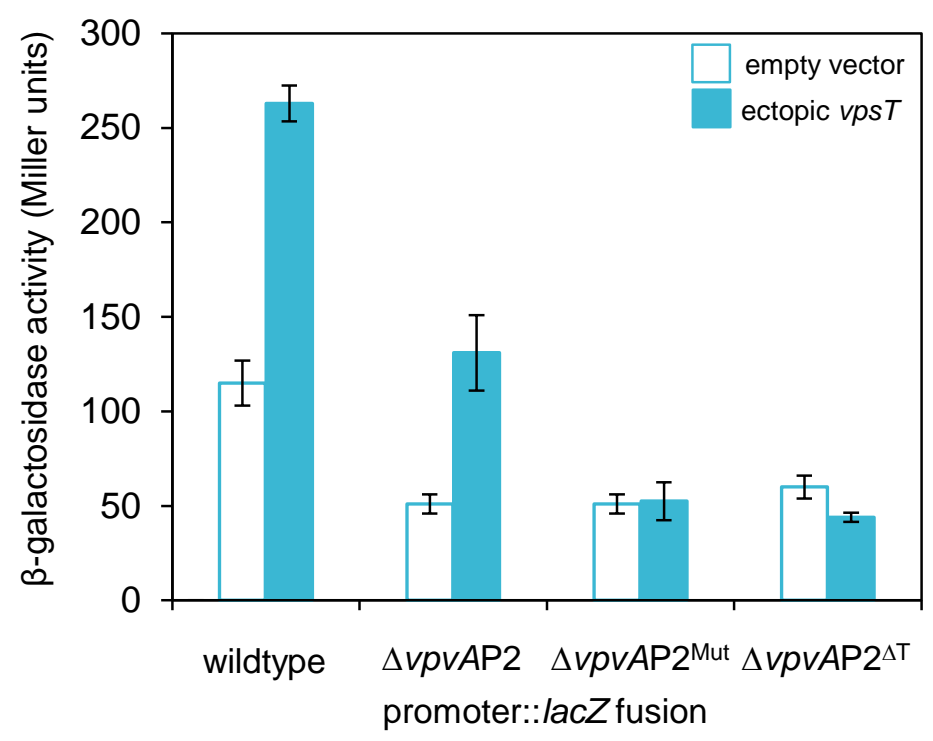




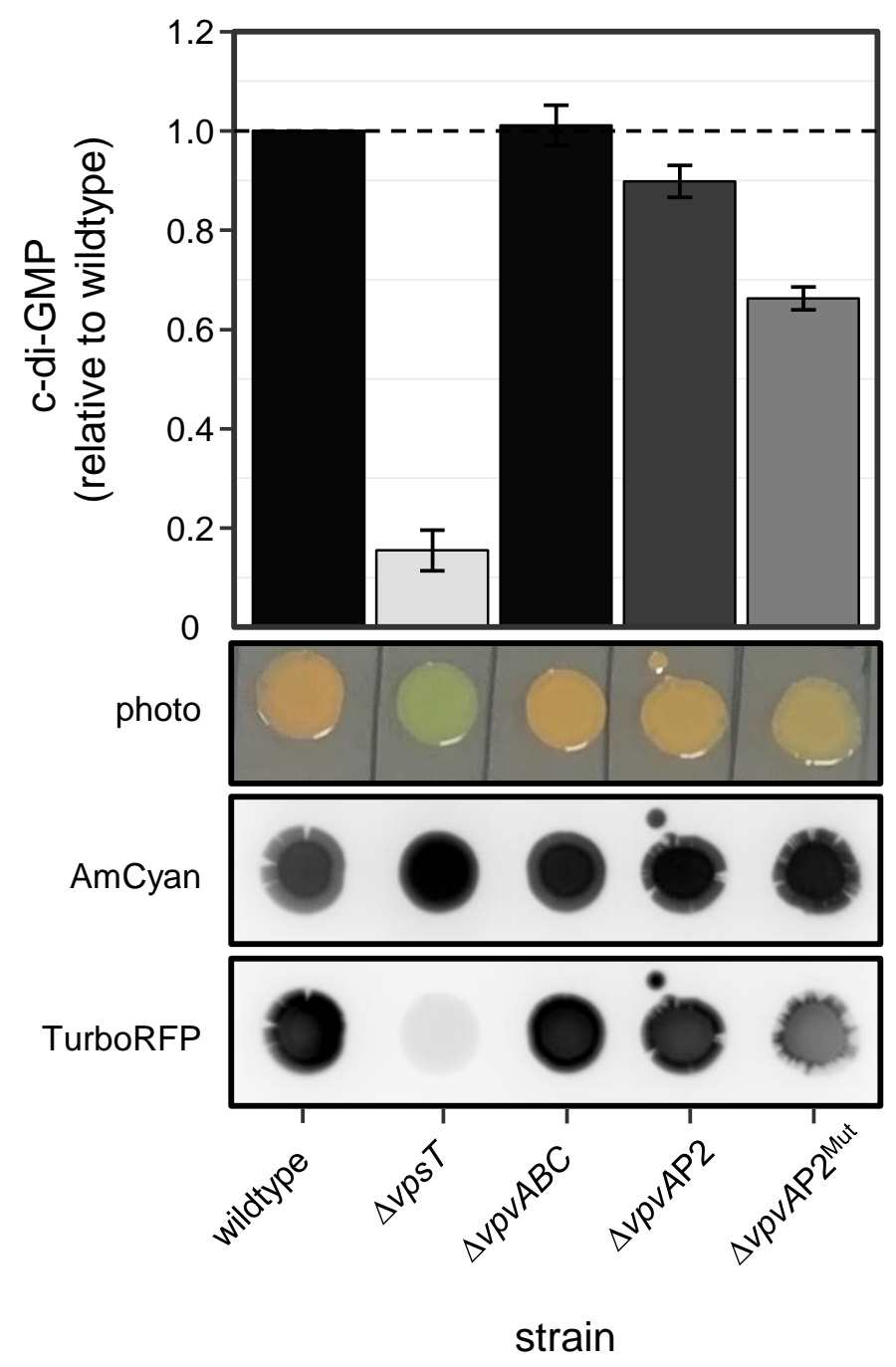




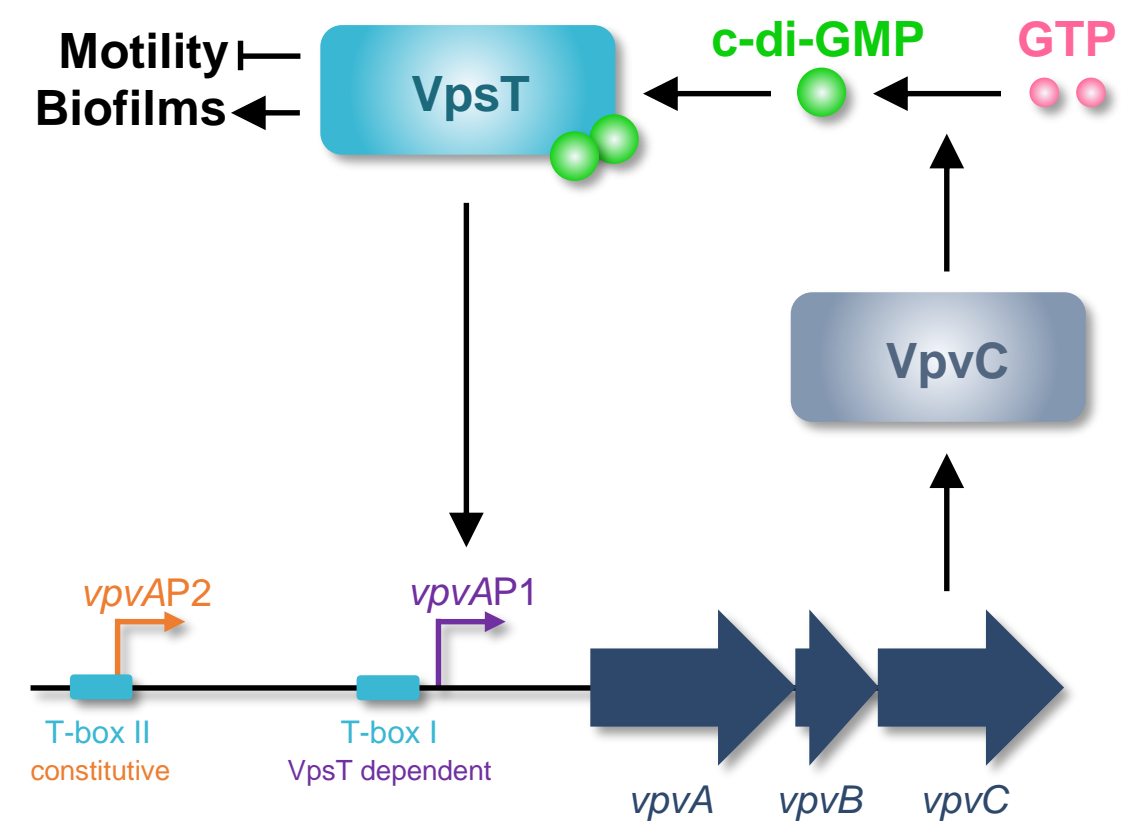




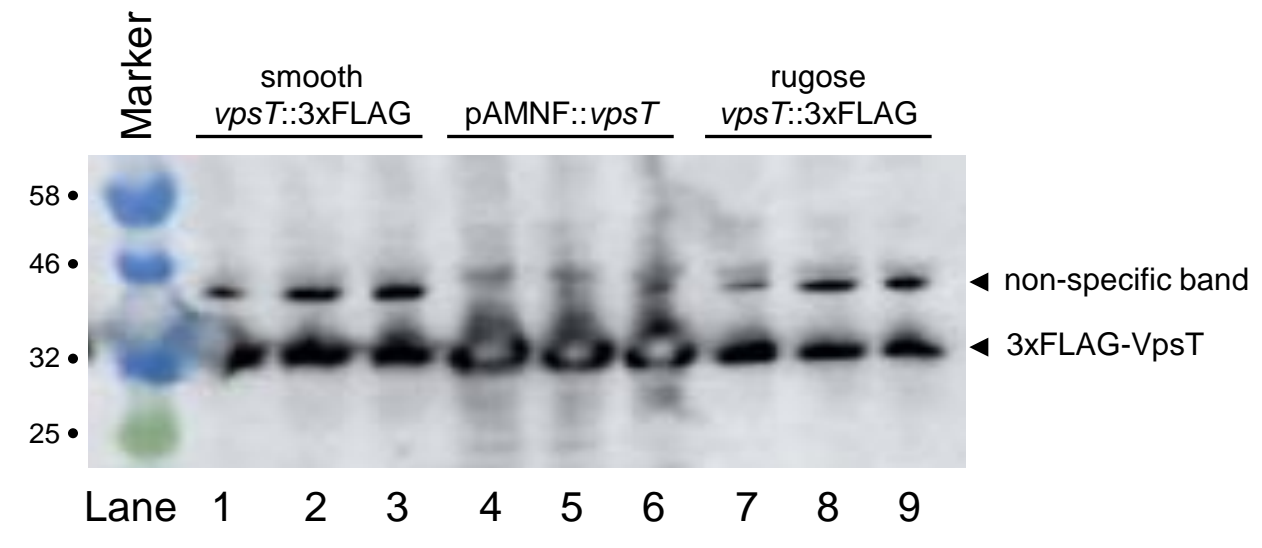




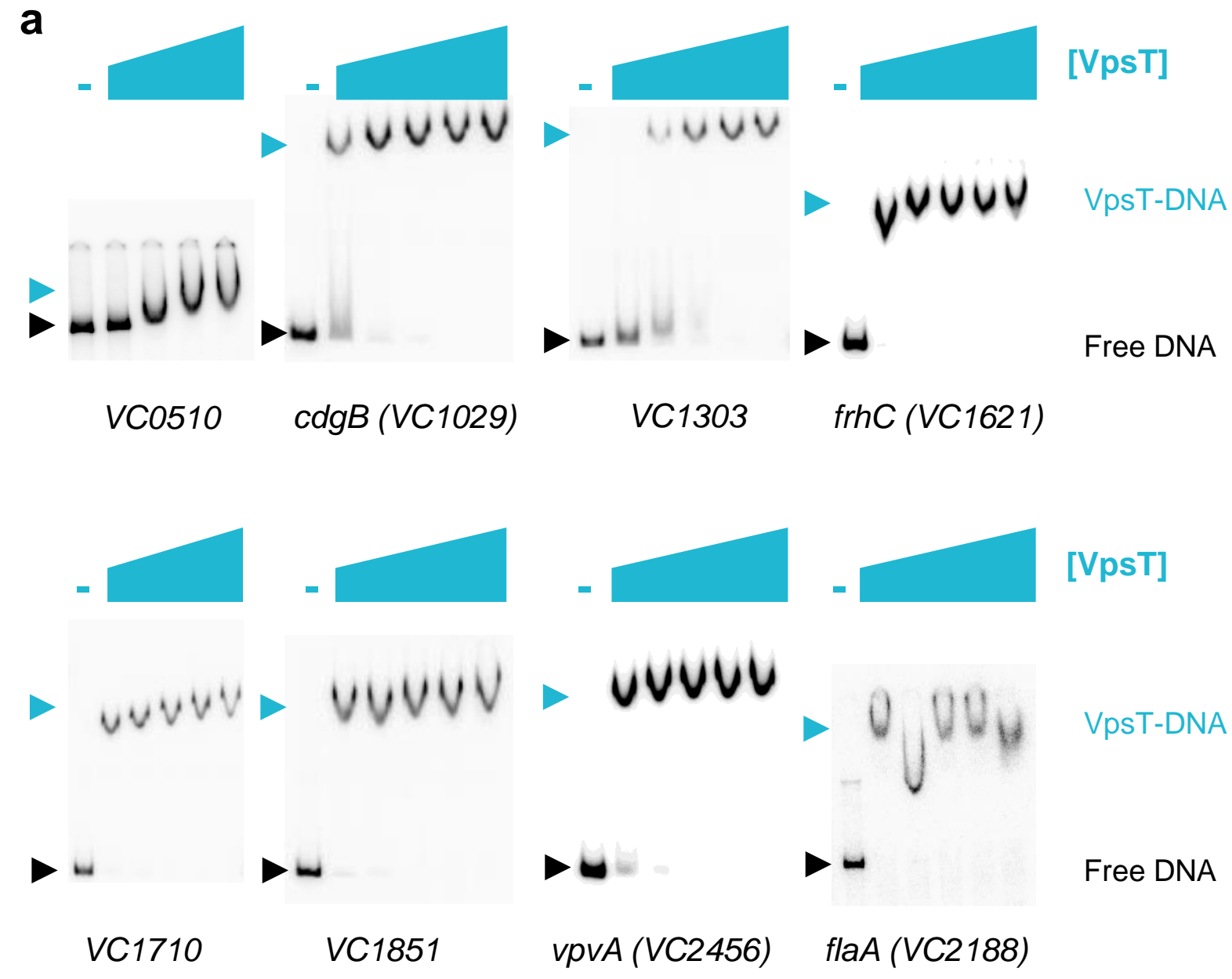

b

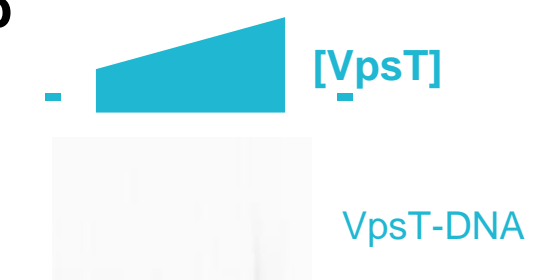

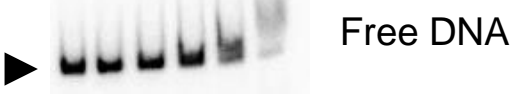

E. coli lacZ 


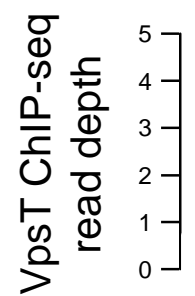

Genes

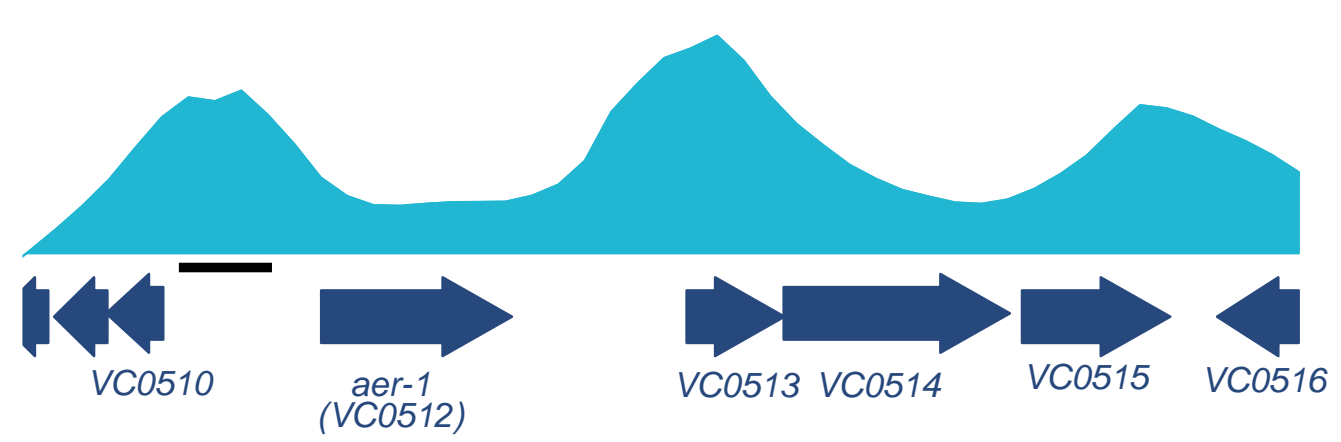

b

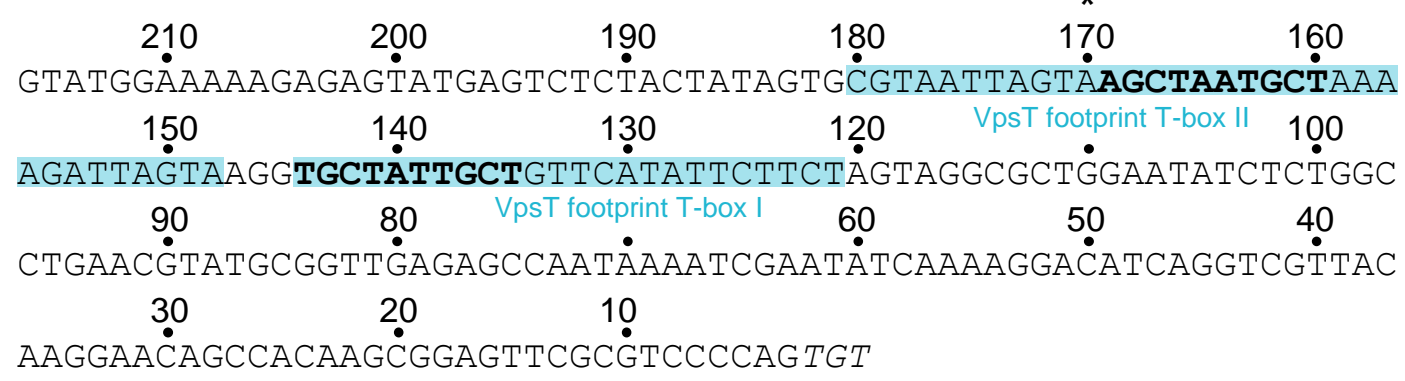

C
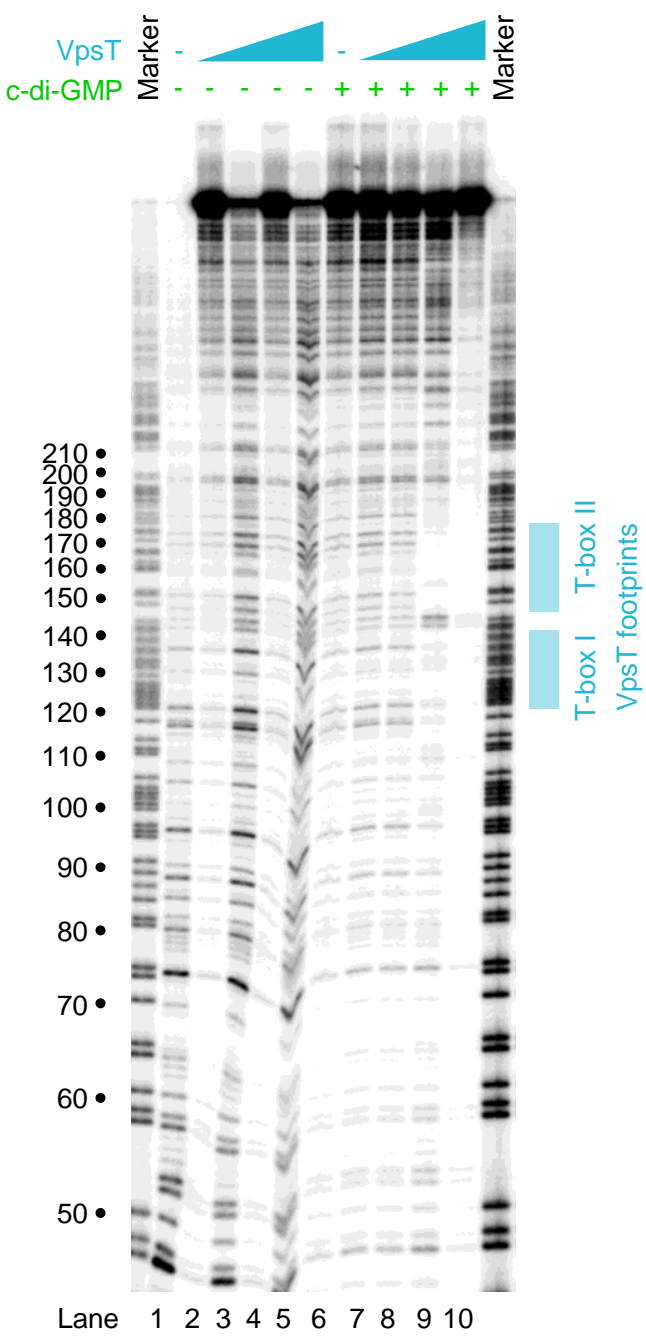
a

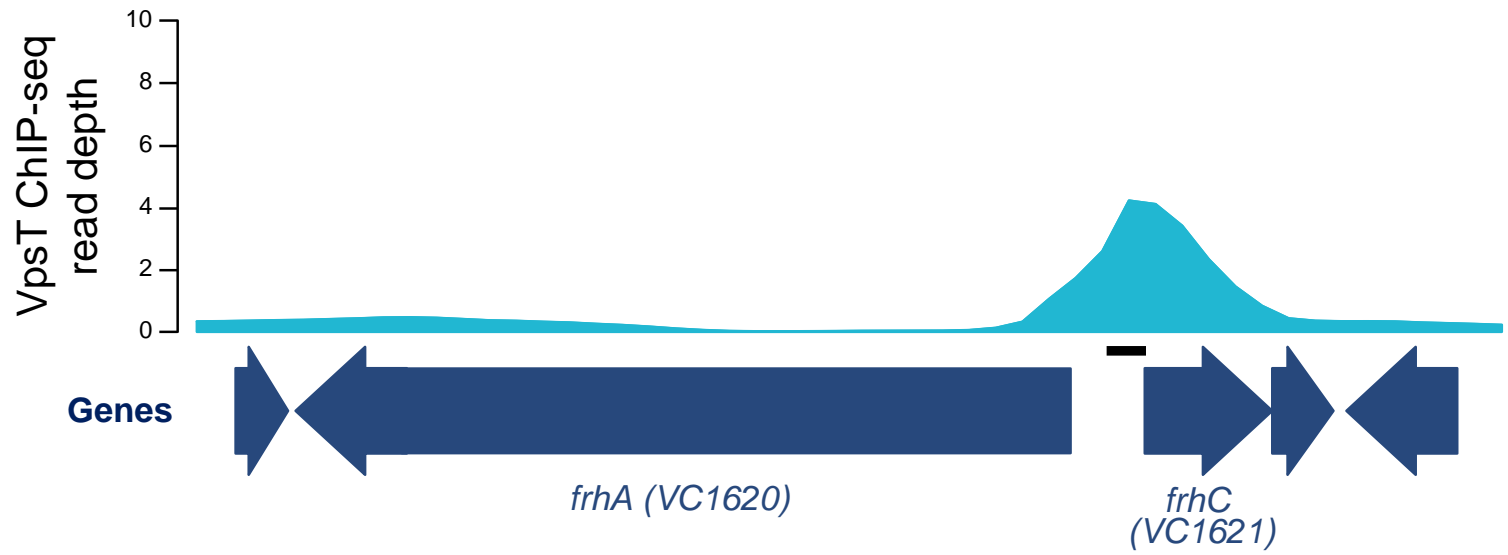

b

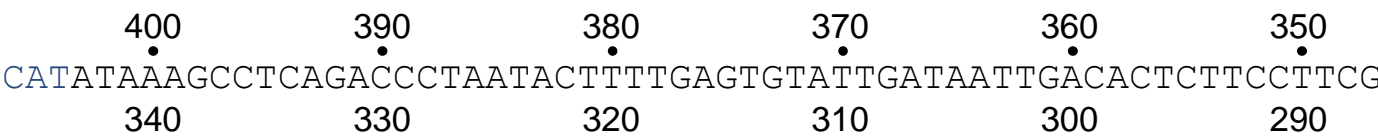

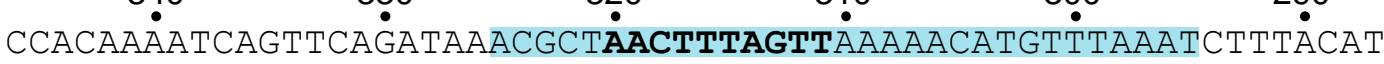
$2802 \quad 270 \quad 260 \quad$ VpsT footprint T-box III $240 \quad 230$ CACAGACGTACCGCTTATGCTGTCTCATCTAGCAACAACATAAATCTTGAACTAAAGTCA $\begin{array}{lllll}220 & 210 & 200 & 190 & \text { VpST footprint T-box II } 170\end{array}$

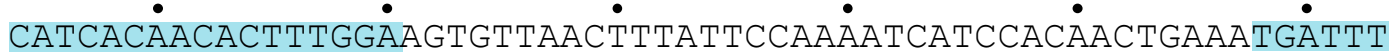
160
150
140
$\star 130$
120
110

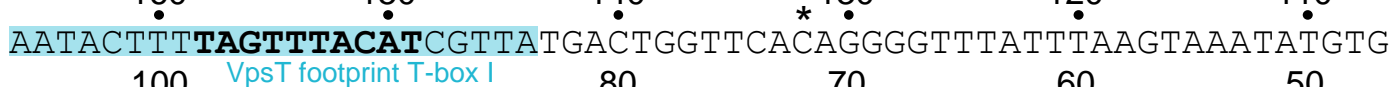
100
$\bullet$
80
70.
60
50

ATACGCTTCTCGCGTGCGTCATATTGTTGACGTCAAATATCCTATATGTCTTATAAATGA

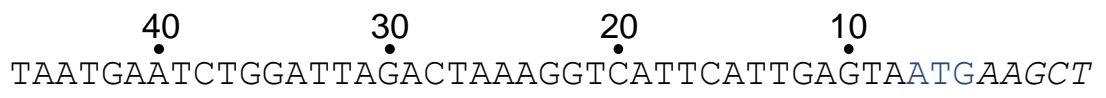

C
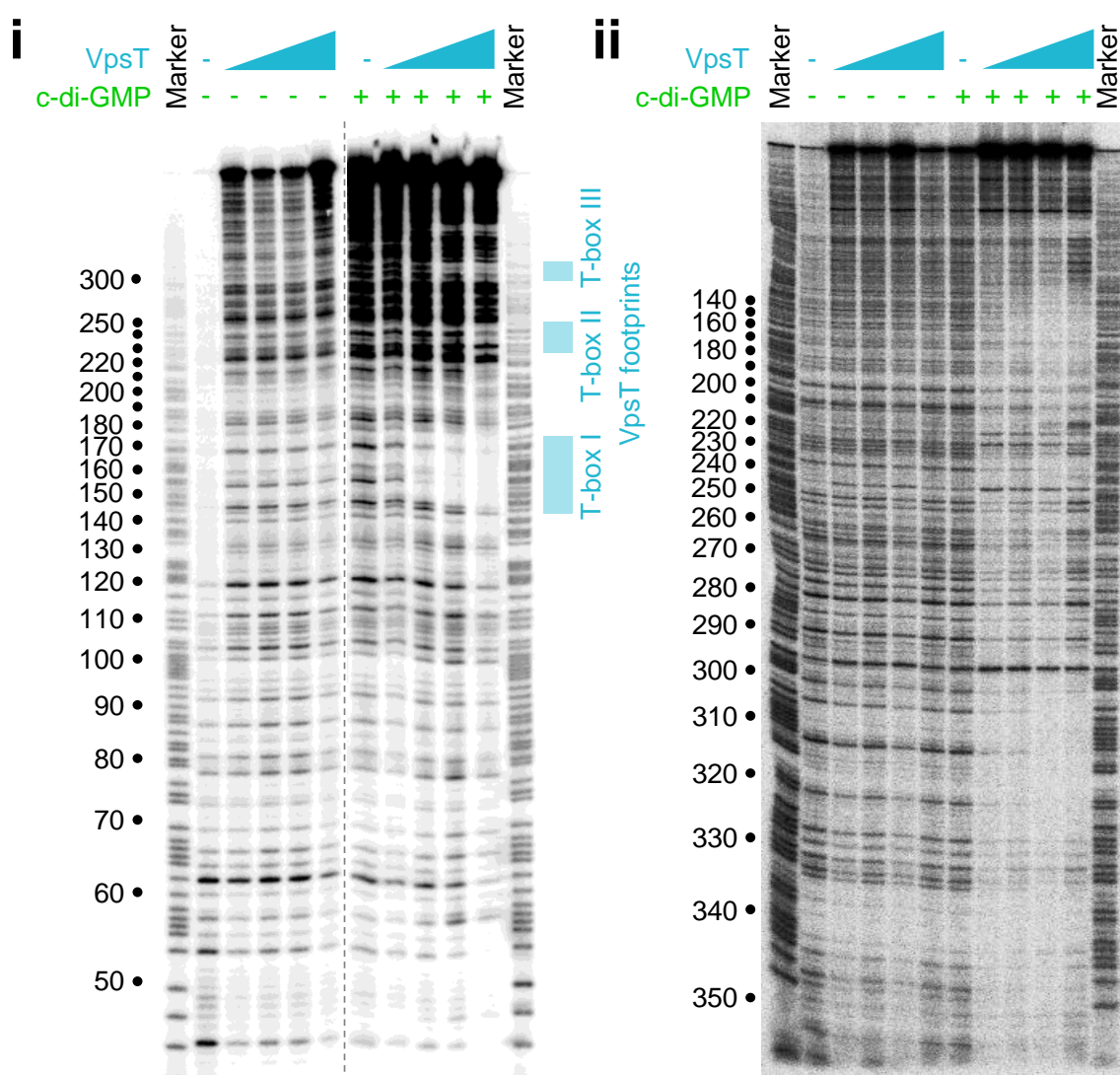

140

180

$200:$

220

250

$260 \bullet$

$270 \bullet$

$280 \bullet$

$290 \bullet$

300 •

310 •

320 •

330 •

340 •

350 •

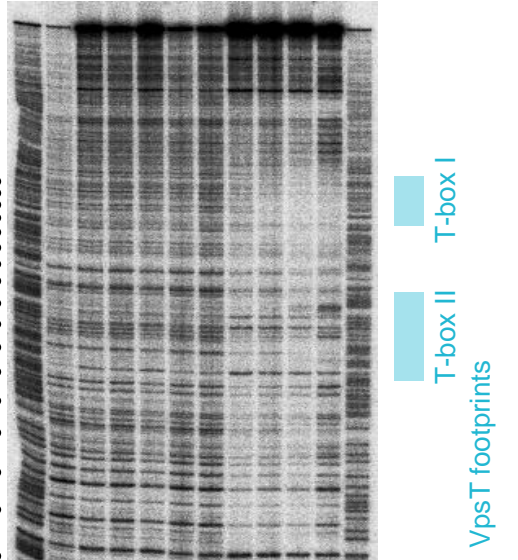

. 
(which was not certified by peer review) is the author/funder, who has granted bioRxiv a license to display the preprint in perpetuity. It is $m$ available under aCC-BY 4.0 International license.

Figure S5

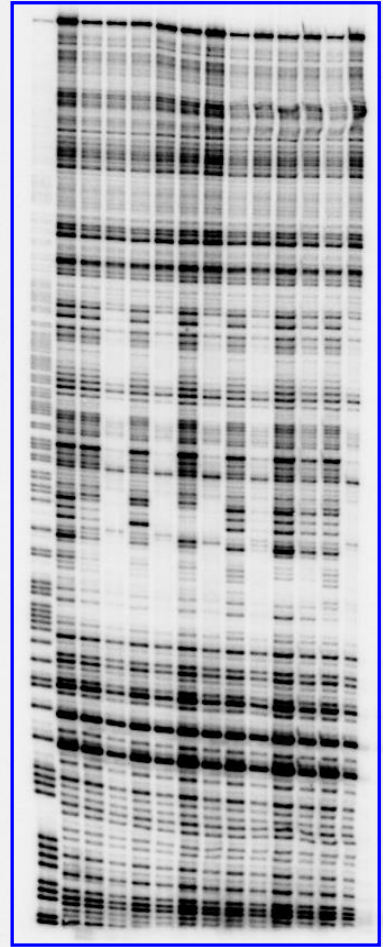

Figure 2

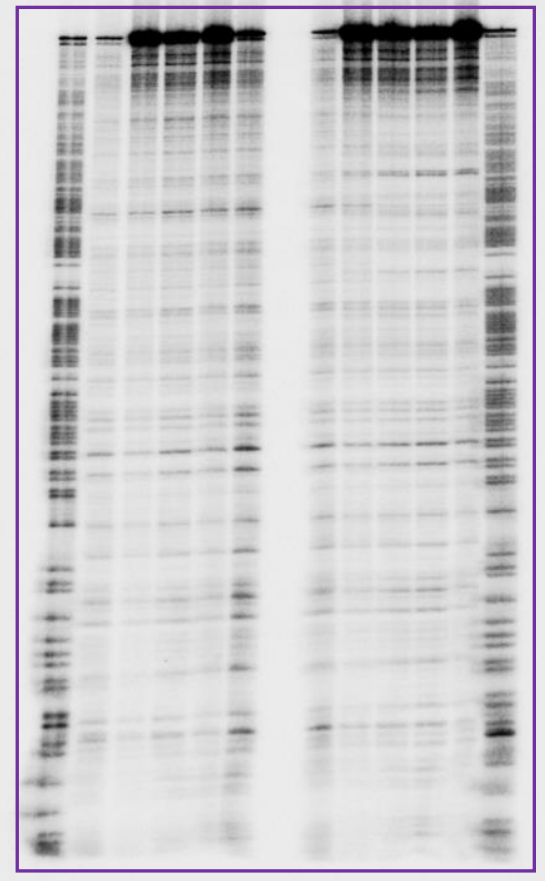

Figure 3c

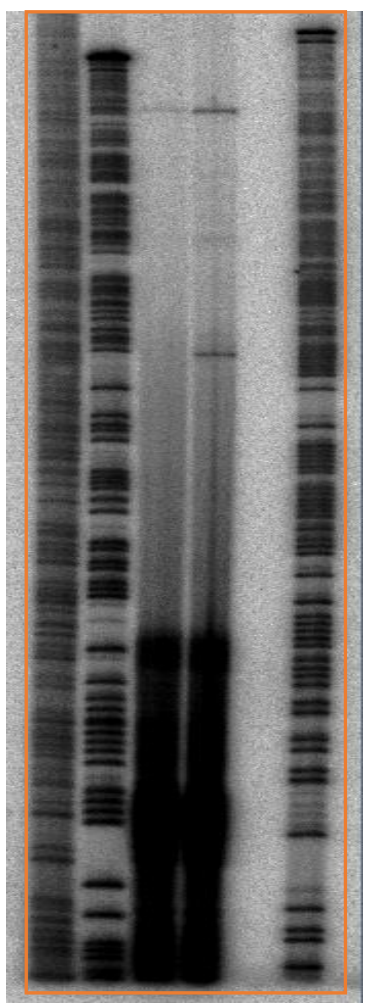

Figure 3d

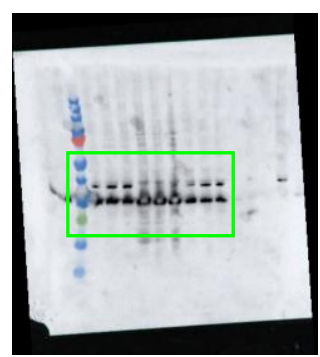

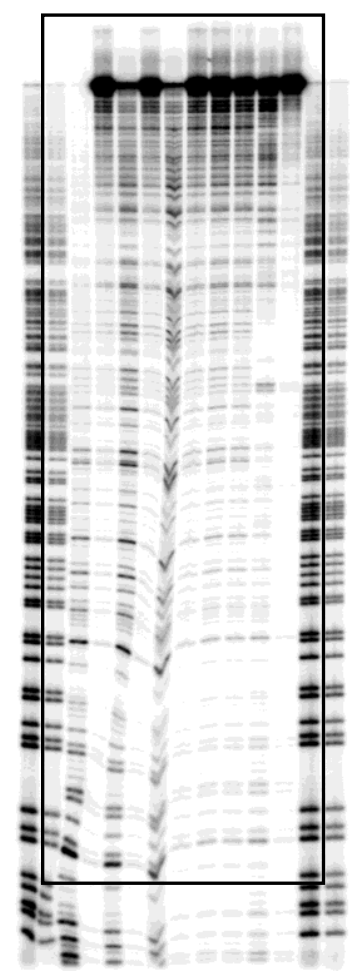

Figure S3
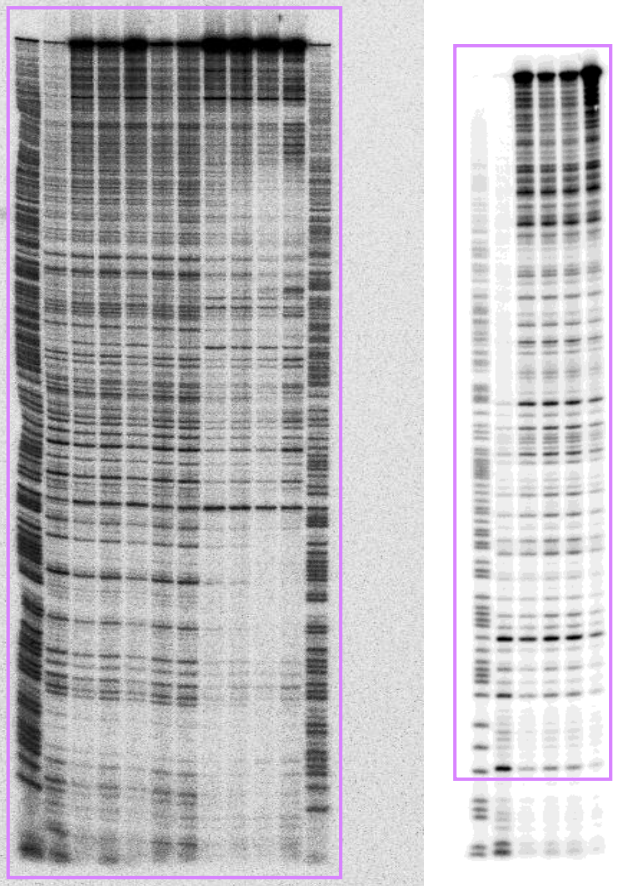

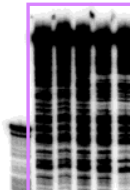

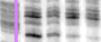

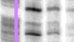

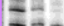

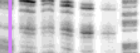

ㄱ․는

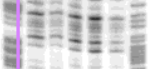

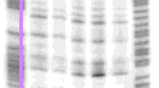

尚

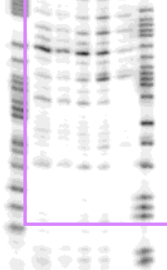


(which was not certified by peer review) is the author/funder, who has granted bioRxiv a license to display the preprint in perpetuity. It is $m$ VC1621 available under aCC-BY 4.0 International license.

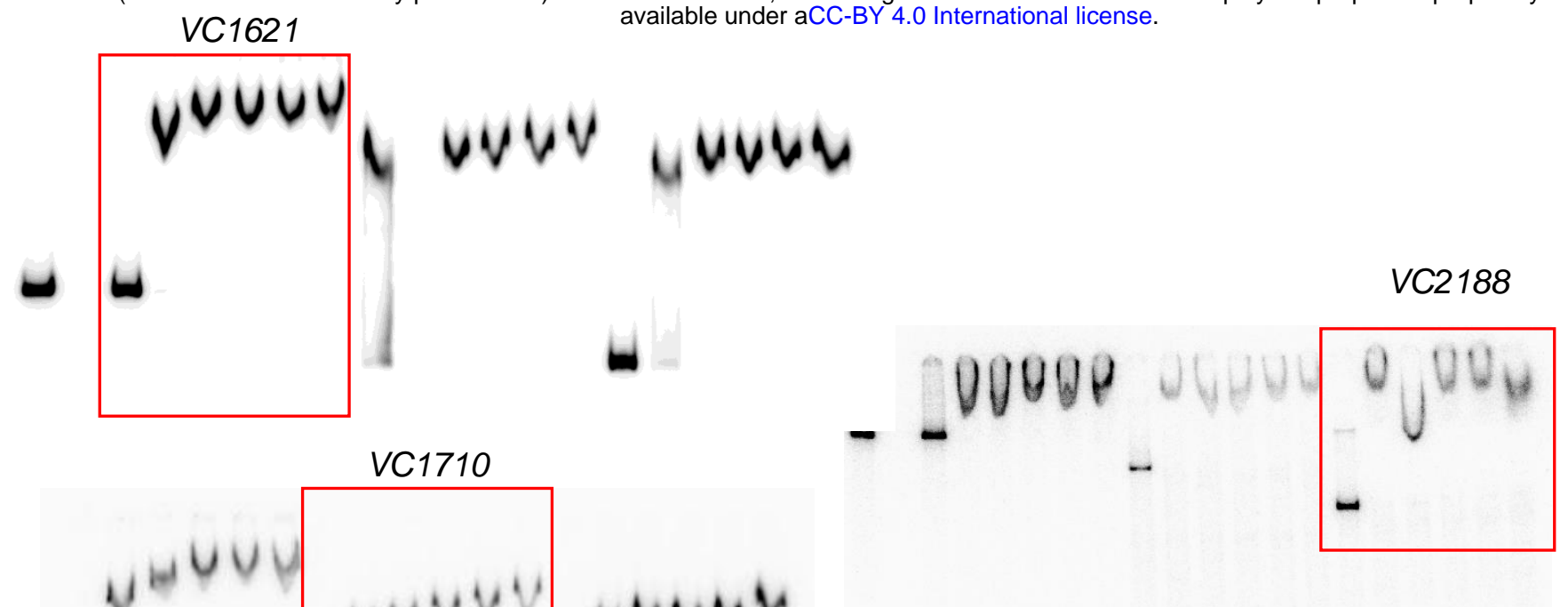

\section{UWVWV UNOWV}
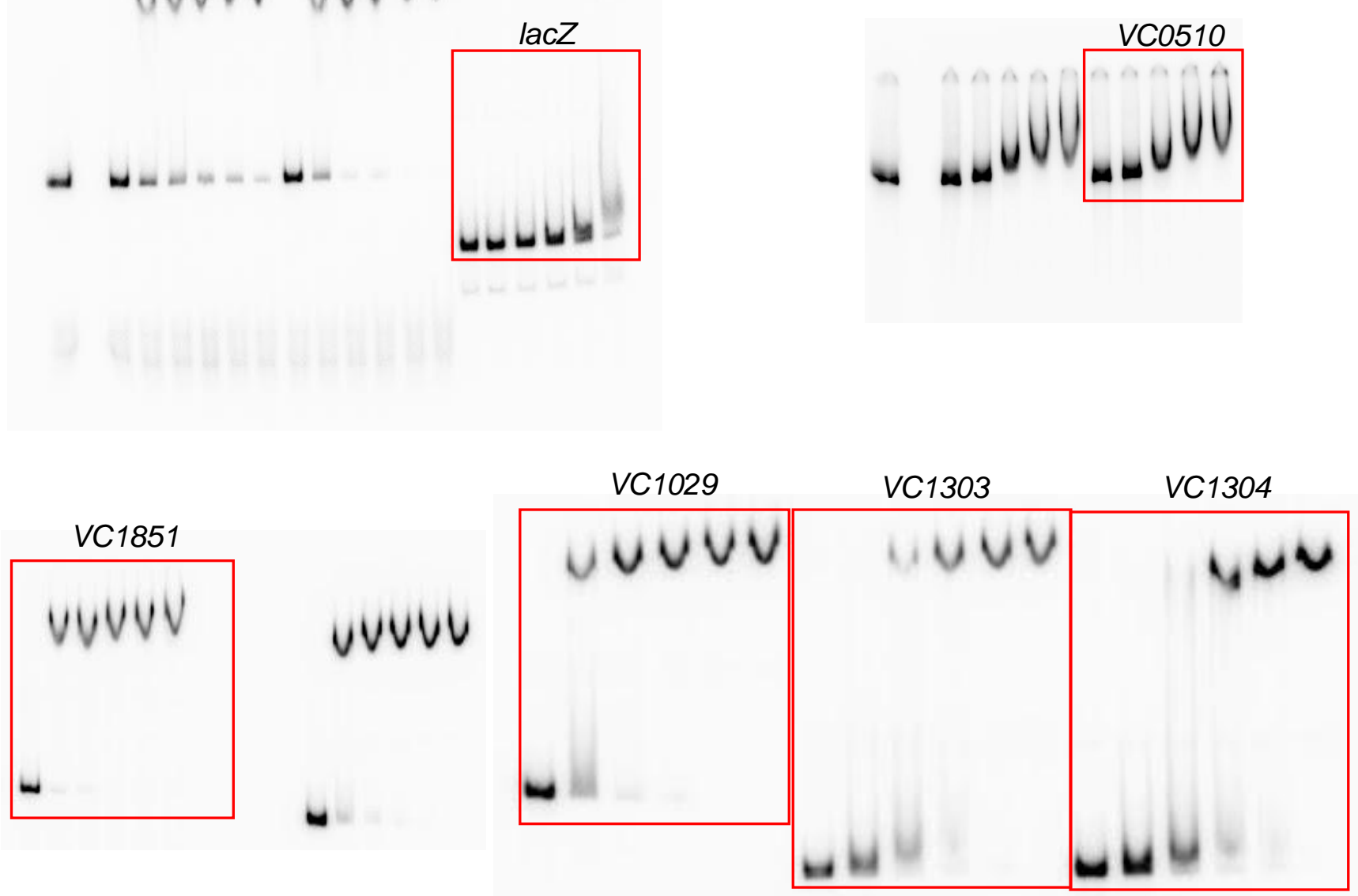

Figure S2 